\title{
Simulation of Hydrogenated Amorphous Silicon: Temperature Dependence of Nonequilibrium Distribution Functions for Trap States
}

\author{
Azuma Suzuki ( $\square$ asuzuki@cl.cilas.net) \\ Research Institute of Photovoltaic, SUNNAN https://orcid.org/0000-0002-8757-1126
}

\section{Research Article}

Keywords: temperature dependence, nonequilibrium distribution function, hopping, amorphous silicon, photoconductivity, thermal quenching

Posted Date: April 27th, 2021

DOI: https://doi.org/10.21203/rs.3.rs-443421/v1

License: (c) (i) This work is licensed under a Creative Commons Attribution 4.0 International License.

Read Full License 


\title{
Simulation of hydrogenated amorphous silicon: temperature dependence of nonequilibrium distribution functions for trap states
}

\author{
Azuma Suzuki \\ Research Institute of Photovoltaic, SUNNAN, 3-17-10-1103 Ougibashi, Kouto-ku, Tokyo 135-0011, Japan \\ E-mail: asuzuki@cl.cilas.net
}

ORCID: 0000-0002-8757-1126

\begin{abstract}
The nonequilibrium distribution functions (NDF) for the trap states in the mobility-gap under photo illumination and zero bias voltage are derived by the constructed self-consistent drift-diffusion simulator consisted of the Poisson equation and current continuity equations for hydrogenated amorphous silicon (a-Si:H). As for the temperature dependence of the NDF, we find that the values of the NDF decrease with increasing temperature (the negative temperature dependence) in the energy region near the conduction band for p-type a-Si:H. That is the reverse of the temperature dependence of the equilibrium distribution functions (EDF) for the trap states in the mobility-gap. Furthermore, we show that the new physical characteristic is applicable to the explanation of the temperature characteristic of the photoconductivity caused by the electron hopping in the conduction band tail for a-Si:H. The photoconductivity of a-Si:H decreases with increasing temperature, which is called the thermal quenching (TQ). We show that the TQ observed in a low temperature around 200K for p-type a-Si:H can be explained by the electron hopping model with the p-type NDF having the negative temperature dependence.
\end{abstract}

\section{Keywords}

temperature dependence, nonequilibrium distribution function, hopping, amorphous silicon, photoconductivity, thermal quenching,

\section{Introduction}

Many theoretical models for the capture and emission processes between the trap states in the mobility-gap and the bands (the valence band (VB) and the conduction band (CB)) have been proposed. Simmons and Taylor [1-4] have proposed the simple model based on the Shockley-Read-Hall recombination model [5]. The key idea of the 
model concept is that the nonequilibrium steady-state condition is applied for each energy state in the mobilitygap. The main advantage of this approach is that the nonequilibrium distribution functions (NDF) for the trapstates in the mobility gap can be uniquely determined. The temperature dependence of the equilibrium distribution functions (EDF) for the trap states in the mobility-gap is well known, that is the same as Fermi-Dirac distribution function. But, the temperature dependence of the NDF has not been argued exactly in the past. The density of trap carriers in the mobility-gap is derived by integrating the product of the NDF and the density of states (DOS) at each trap energy for the interval the VB to the CB. Therefore, the temperature dependence of the trap carriers' density in the mobility-gap is determined by that of the NDF. That is extremely important for the argument of the temperature dependence of some physical phenomenon concerned with the electron density in the conduction band tail for a-Si:H. We study the temperature dependence of the NDF under photo illumination and zero bias voltage rigorously and find a new characteristic. Furthermore, we try to show that the new physical characteristic is applicable to the explanation of the temperature characteristic of the photoconductivity caused by the electron hopping [6-9] in the conduction band tail for a-Si:H.

Figure 1 is the typical measured photoconductivity of a-Si:H for p-type, i-type, and n-type as a function of temperature $[10,11]$. In the area surrounded by broken line, the photoconductivity turns over from the increase to the decrease with increasing temperature: the temperature coefficient of the photoconductivity turns over from the positive to the negative. Further, the photoconductivity turns over from the decrease to the increase with more increasing temperature. This temperature characteristic of the photoconductivity is called the thermal quenching (TQ). Physical explanations of the TQ in a-Si:H have been reported by many researches [10-21]. Tran assumed that the recombination center changed from the VB tail to the dangling bond (DB) with increasing temperature, and the capture rate of the DB was larger than that of the VB tail. But the quantitative fit of the temperature dependence of the photoconductivity with experimental data had to be explained by hopping processes [12]. Merazga followed the approach of Tran; he assumed that the capture rate of the DB was larger than that of the VB tail, and included the process of the electron hopping in the CB tail states [13]. But, Merazga calculated the density of the hopping carriers in the steady state quasi-equilibrium despite the nonequilibrium condition under photoirradiation. As regards that matter, we treat the nonequilibrium capture and emission process rigorously. We study the degree of effect of the electron hopping for the photoconductivity and we find that the TQ can be explained by the electron hopping in a low temperature. That is the TQ in a low temperature can be explained by the temperature dependence of the NDF only.

As a supplemental explanation, the aim of this study is the study of the temperature dependence of the NDF for p-type and n-type a-Si:H and to show that the new physical property can be applied to the explanation of the TQ caused by the electron hopping in the conduction band tail in a low temperature around $200 \mathrm{~K}$, but not to reproduce the data of Fig. 1 accurately. The dark conductivity is orders of magnitude smaller than the photoconductivity so that it can be neglected.

This paper is organized as follows. Section 2 introduces the theoretical model and the numerical simulation method. In Sec. 3, we present the simulation results of the temperature dependence of the NDF, the TQ of the photoconductivity, and their physical interpretation. Finally, we describe conclusions in Sec. 4. 


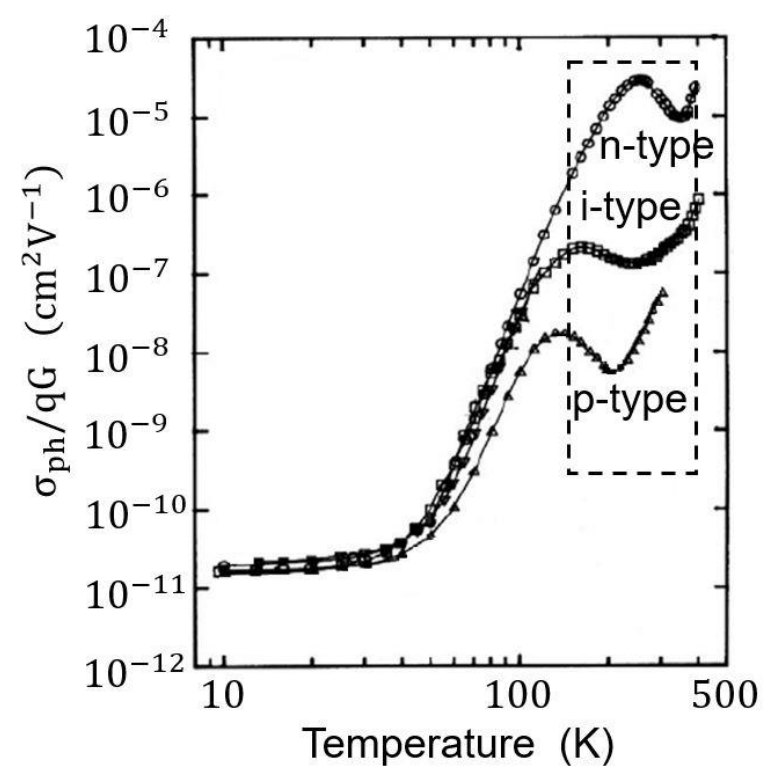

Fig. 1 The typical measured photoconductivity in a-Si:H for p-type, i-type and n-type as a function of temperature $[10,11]$. In the area surrounded by broken line, the photoconductivity turns over from the increase to the decrease with increasing temperature. Further, the photoconductivity turns over from the decrease to the increase with more increasing temperature. This temperature characteristic is called the thermal quenching (TQ).

\section{Numerical method}

\subsection{Capture and emission processes}

Regarding the capture and emission processes, we follow the approaches taken by Hack [22-25], Fantoni [26,27] and Walker [28], which consider the Simmons and Taylor model [1-4]. As for the Shockley-Read-Hall model, two capture and two emission processes are considered for each trap state, which are shown in Fig. 2. The transition $a^{A}$ is an electron capture process from the $\mathrm{CB}$ via the acceptor-like trap states located at energy $E$ and $b^{A}$ is an electron emission process from that states to the $\mathrm{CB}$. The transition $c^{A}$ is a hole capture process from the $\mathrm{VB}$ via the acceptor-like trap states located at energy $E$ and $d^{A}$ is a hole emission process from that states to the VB. The solid line arrows and broken line arrows indicate the transition directions of the electrons and the holes, respectively in Fig. 2. Similarly, four capture and emission processes of the electrons and the holes via the donor-like trap states located at energy $E$ are also shown in Fig. 2: $a^{D}, b^{D}, c^{D}$, and $d^{D}$. We assume that the capture and emission processes at the different energy $E$ in the mobility gap do not have a relationship to each other. Then, by employing the Shockley-Read-Hall model, the electron capture and the electron emission rates per unit volume for the acceptor-like trap states are expressed as follows:

$$
r_{a^{A}}=v_{t h} \sigma_{n}^{A} n N_{t}^{A}\left(1-f^{A}(E)\right),
$$




$$
r_{b^{A}}=v_{t h} \sigma_{n}^{A} N_{C} \exp \left(\frac{E-E_{C}}{k_{B} T}\right) N_{t}^{A} f^{A}(E),
$$

where, $n$ is the density of electrons in the $\mathrm{CB}, N_{t}^{A}$ is the density of acceptor-like trap, $E_{C}$ is the energy of the CB edge, $k_{B}$ is the Boltzmann constant, and $T$ is the system temperature. $v_{t h}, \sigma_{n}^{A}$ and $N_{C}$ are described in the Table 1. $f^{A}(E)$ is the function of the NDF for the acceptor-like trap states, which is the different from the Fermi-Dirac distribution function under photo-illumination in general. Likewise, the capture and emission rates per unit volume for holes are expressed as follows:

$$
\begin{gathered}
r_{c^{A}}=v_{t h} \sigma_{p}^{A} p N_{t}^{A} f^{A}(E), \\
r_{d^{A}}=v_{t h} \sigma_{p}^{A} N_{V} \exp \left(\frac{E_{V}-E}{k_{B} T}\right) N_{t}^{A}\left(1-f^{A}(E)\right),
\end{gathered}
$$

where $p$ is the density of holes in the VB and $E_{v}$ is the energy of the VB edge. $\sigma_{p}^{A}$ and $N v$ are described in the Table 1.

As for the capture and the emission rates per unit volume of the electrons and the holes via the donor-like trap states are expressed by changing suffix "A" to "D"; $r_{a^{D}}, r_{b^{D}}, r_{c^{D}}$, and $r_{d^{D}}$ correspond to the capture and emission processes $a^{D}, b^{D}, c^{D}$, and $d^{D}$, respectively.

Under the nonequilibrium steady-state condition at each trap state, the formula for the acceptor-like trap states and the donor-like trap states are shown as follows:

$$
\begin{gathered}
r_{a^{A}}-r_{b^{A}}=r_{c^{A}}-r_{d^{A}}, \\
r_{a^{D}}-r_{b^{D}}=r_{c^{D}}-r_{d^{D}} .
\end{gathered}
$$

The formula of the NDF for the acceptor-like trap states is derived from Eq. (5) and it is expressed as follows:

$$
f^{A}(E)=\frac{n+C^{A} N_{V} \exp \left(\frac{E_{V}-E}{k_{B} T}\right)}{n+N_{C} \exp \left(\frac{E-E_{C}}{k_{B} T}\right)+C^{A}\left(p+N_{V} \exp \left(\frac{E_{V}-E}{k_{B} T}\right)\right)} \quad: C^{A}=\frac{\sigma_{p}^{A}}{\sigma_{n}^{A}}
$$

The formula of the NDF for the donor-like trap states is derived from Eq. (6) and it is expressed as follows:

$$
f^{D}(E)=\frac{C^{D} n+N_{V} \exp \left(\frac{E_{V}-E}{k_{B} T}\right)}{C^{D}\left(n+N_{C} \exp \left(\frac{E-E_{C}}{k_{B} T}\right)\right)+p+N_{V} \exp \left(\frac{E_{V}-E}{k_{B} T}\right)} \quad: C^{D}=\frac{\sigma_{n}^{D}}{\sigma_{p}^{D}}
$$


When trap energy level $E$ is far away from $E_{V}$ and $E_{C}\left(E_{V} \ll E \ll E_{C}\right)$, the electron and hole emission rates become very small; $\exp \left(\frac{E_{V}-E}{k_{B} T}\right) \cong 0$ and $\exp \left(\frac{E-E_{C}}{k_{B} T}\right) \cong 0$ are satisfied. Then Eq. (9) and Eq. (10) are derived from Eq. (7) and Eq. (8), respectively,

$$
\begin{aligned}
& f^{A}(E)=\frac{n}{n+C^{A} p}, \\
& f^{D}(E)=\frac{C^{D} n}{C^{D} n+p} ;
\end{aligned}
$$

the NDF have the constant values determined by band carriers.

When the trap states exist successively in the mobility-gap, the total capture and emission rates per unit volume are calculated by integrating the capture and emission rates per unit volume at each energy level for the interval the VB to the CB, which has been proposed by Simmons and Taylor [1-4]. That is expressed as follows for the acceptorlike states; $R_{a^{A}}, R_{b^{A}}, R_{c^{A}}$, and $R_{d^{A}}$ correspond to the capture and emission processes $a^{A}, b^{A}, c^{A}$, and $d^{A}$, respectively. $g_{T}^{A}(E)$ and $g_{D}^{A}(E)$ are the DOS of a-Si:H in the mobility-gap, which are shown in the next section.

$$
\begin{aligned}
& R_{a^{A}}=\int_{E_{V}}^{E_{C}} v_{t h} \sigma_{n}^{A} n\left(g_{T}^{A}(E)+g_{D}^{A}(E)\right)\left(1-f^{A}(E)\right) d E \\
& R_{b^{A}}=\int_{E_{V}}^{E_{C}} v_{t h} \sigma_{n}^{A} N_{C} \exp \left(\frac{E-E_{C}}{k_{B} T}\right)\left(g_{T}^{A}(E)+g_{D}^{A}(E)\right) f^{A}(E) d E \\
& R_{c^{A}}=\int_{E_{V}}^{E_{C}} v_{t h} \sigma_{p}^{A} p\left(g_{T}^{A}(E)+g_{D}^{A}(E)\right) f^{A}(E) d E \\
& R_{d^{A}}=\int_{E_{V}}^{E_{C}} v_{t h} \sigma_{p}^{A} N_{V} \exp \left(\frac{E_{V}-E}{k_{B} T}\right)\left(g_{T}^{A}(E)+g_{D}^{A}(E)\right)\left(1-f^{A}(E)\right) d E .
\end{aligned}
$$

As for the donor-like states, the total capture and emission rates per unit volume are expressed by changing suffix "A" to "D"; $R_{a^{D}}, R_{b^{D}}, R_{c^{D}}$, and $R_{d^{D}}$ correspond to the capture and emission processes $a^{D}, b^{D}, c^{D}$, and $d^{D}$, respectively. 


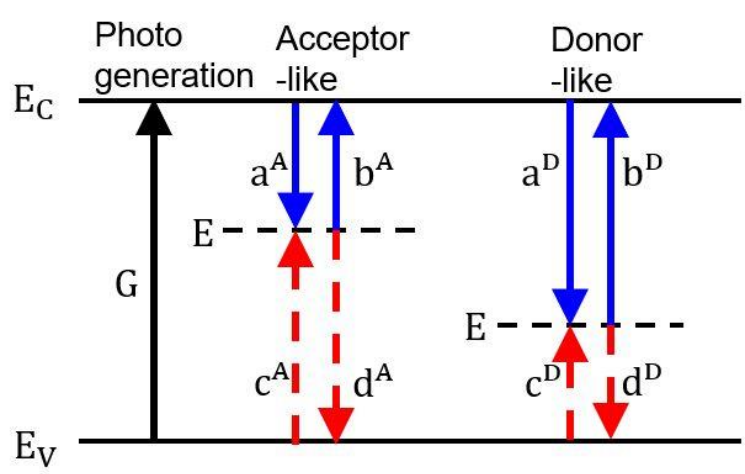

Fig. 2 Two capture and two emission processes of the electrons and the holes via the acceptor-like trap states located at energy $E\left(a^{A}, b^{A}, c^{A}\right.$, and $\left.d^{A}\right)$ and those via the donor-like trap states located at energy $E\left(a^{D}, b^{D}, c^{D}\right.$, and $d^{D}$ ) under photo-illumination. The solid line arrows and the broken line arrows indicate the transition directions of the electrons and the holes, respectively. The electron photo generation process $G$ is also shown.

\subsection{Density of states of a-Si:H}

As for the density of states (DOS) in the mobility-gap for a-Si:H, we employ the tail states and the deep states [24,26,29-32]. The tail states caused by the material disorder are modeled with the exponential tail from the CB edge or the VB edge. In the present study, the tail states DOS are modeled as follows: the acceptor-like $g_{T}^{A}(E)$ and the donor-like $g_{T}^{D}(E)$.

$$
\begin{aligned}
& g_{T}^{A}(E)=g_{0} \exp \left(\frac{E-E_{m}}{E_{A}}\right), \\
& g_{T}^{D}(E)=g_{0} \exp \left(\frac{E_{m}-E}{E_{D}}\right),
\end{aligned}
$$

where $E_{m}, g_{0}, E_{A}$, and $E_{D}$ are described in the Table 1. On the other hand, the deep states associated with the $\mathrm{DB}$ of a-Si:H are modeled by the Gaussian distribution with respect to energy. The DOS of the acceptor-like deep states $g_{D}^{A}(E)$ and that of the donor-like deep states $g_{D}^{D}(E)$ are expressed as follows:

$$
\begin{aligned}
& g_{D}^{A}(E)=\frac{N_{g}^{A}}{\sigma_{A}} \exp \left(-\frac{\left(E-E_{g}^{A}\right)^{2}}{2 \sigma_{A}{ }^{2}}\right) \\
& g_{D}^{D}(E)=\frac{N_{g}^{D}}{\sigma_{D}} \exp \left(-\frac{\left(E-E_{g}^{D}\right)^{2}}{2 \sigma_{D}{ }^{2}}\right)
\end{aligned}
$$

where $E_{g}^{A}, E_{g}^{D}, \sigma_{A}, \sigma_{D}, N_{g}^{A}$, and $N_{g}^{D}$ are described in the Table 1. 


\subsection{Drift-diffusion simulation model}

Under the drift-diffusion simulations method, we solve the Poisson equation and the current continuity equations for the electrons and the holes self-consistently. The Poisson equation is expressed as follows:

$$
\frac{d^{2} \varphi}{d x^{2}}=-\frac{q}{\varepsilon}\left(p-n+p_{h}-n_{h}+p_{t}-n_{t}+N_{D}^{+}-N_{A}^{-}\right)
$$

where $\varphi$ is the electrostatic potential, $\varepsilon$ is the dielectric constant of a-Si, $q$ is the elementary charge, $N_{D}^{+}$and $N_{A}^{-}$ are the densities of ionized donor and acceptor, respectively. The densities of trapped electrons and holes in the tail states are denoted by $n_{h}$ and $p_{h}$, respectively. The densities of trapped electrons and holes in the DB are denoted by $n_{t}$ and $p_{t}$, respectively. $n_{h}, p_{h}, n_{t}$, and $p_{t}$ are calculated as follows:

$$
\begin{aligned}
& n_{h}=\int_{E_{V}}^{E_{C}} g_{T}^{A}(E) f^{A}(E) d E, \\
& p_{h}=\int_{E_{V}}^{E_{C}} g_{T}^{D}(E)\left(1-f^{D}(E)\right) d E, \\
& n_{t}=\int_{E_{V}}^{E_{C}} g_{D}^{A}(E) f^{A}(E) d E, \\
& p_{t}=\int_{E_{V}}^{E_{C}} g_{D}^{D}(E)\left(1-f^{D}(E)\right) d E .
\end{aligned}
$$

Under steady state, the current continuity equations for the electrons and the holes are expressed as follows:

$$
\begin{gathered}
-\frac{1}{q} \frac{d J_{n}}{d x}=G-R_{a^{A}}+R_{b^{A}}-R_{a^{D}}+R_{b^{D}} \\
\frac{1}{q} \frac{d J_{p}}{d x}=G-R_{c^{A}}+R_{d^{A}}-R_{c^{D}}+R_{d^{D}}
\end{gathered}
$$

where $J n$ is the electron current density and $J p$ is the hole current density. The electron-hole pairs are generated under photo-illumination; $G$ is the generation rate per unit volume. The current density consists of the drift and diffusion current densities; $J n$ and $J p$ are expressed as follows with the electron (hole) mobility $(\mu n(\mu p))$ and the electron (hole) diffusion coefficient (Dn (Dp)) in the CB (VB). 


$$
\begin{aligned}
& J_{n}=-q \mu_{n} n \frac{d \psi}{d x}+q D_{n} \frac{d n}{d x} \\
& J_{p}=-q \mu_{p} p \frac{d \psi}{d x}-q D_{p} \frac{d p}{d x} .
\end{aligned}
$$

It is assumed that the incident photons totally reflect on the inner surface of the device and make m-times round trips. Under photo-illumination, the electron-hole pair generation rate per unit volume $G(x)$ is expressed as follows:

$$
G(x)=\alpha \Phi \sum_{n=1}^{m}\left(e^{-\alpha(2(n-1) L+x)}+e^{-\alpha(2 n L-x)}\right)
$$

where $L$ is the length of the device, $x$ is the distance from the top of device. We assume that the absorption coefficient $\alpha$ is constant against the energy and $\Phi$ is the incident photon flux, which is the approximation employed by Hack [24].

\subsection{Hopping model}

According to Mott's hopping model, the trap state electron hopping occurs from the initial state at the energy level $E_{i}$ to the destination state at the energy level $E_{j}[6-9,13]$. The single hopping rate $\Gamma_{i, j}\left(\sec ^{-1}\right)$ is calculated under the condition of the energy level $E_{i}$ and $E_{j}$ as follows:

$$
\begin{gathered}
\text { if } E_{i} \geq E_{j} \quad \Gamma_{i, j}=v_{0}\left(\frac{g_{T}^{A}\left(E_{j}\right) d E G}{G T_{i}}\right) \exp \left(-\frac{2 R_{i, j}}{a}\right), \\
G T_{i}=\int_{E_{V}}^{E_{i}} g_{T}^{A}(E) d E, \\
R_{i, j}=\left(\frac{4 \pi}{3} G T_{i}\right)^{-1 / 3}, \\
\text { if } E_{i}<E_{j} \quad \Gamma_{i, j}=v_{0}\left(\frac{g_{T}^{A}\left(E_{i}\right) d E G}{G T_{j}}\right) \exp \left(-\frac{2 R_{i, j}}{a}\right) \exp \left(-\frac{E_{j}-E_{i}}{k_{B} T}\right), \\
G T_{j}=\int_{E_{V}}^{E_{j}} g_{T}^{A}(E) d E,
\end{gathered}
$$




$$
R_{i, j}=\left(\frac{4 \pi}{3} G T_{j}\right)^{-1 / 3},
$$

where $v_{0}, a$ and $d E G$ are described in Table 1. $g_{T}^{A}(E)$ is Eq. (15). $\frac{g_{T}^{A}\left(E_{j}\right) d E G}{G T_{i}}$ in Eq. (29) and $\frac{g_{T}^{A}\left(E_{i}\right) d E G}{G T_{j}}$ in Eq. (32) are the weight coefficients of the hopping probability for $E_{i} \geq E_{j}$ and $E_{i}<E_{j}$, respectively. $G T_{i}$ and $G T_{j}$ are the total DOS of the tail states under the energy level $E_{i}$ and $E_{j}$, respectively. $R_{i, j}$ is the hopping length from the initial state at the energy level $E_{i}$ to the destination state at the energy level $E_{j}$. The summation of the hopping rates per unit volume for the trapped electrons in the acceptor-like band tail $\left(R_{h o, i}^{n}\right)$, which is the hopping from the initial states at the energy level $E_{i}$ to a lot of destination states at the energy level $E_{j}$, is calculated with the single hopping rate $\Gamma_{i, j}$ as follows:

$$
\begin{gathered}
R_{h o, i}^{n}=n h_{i} \sum_{j} \Gamma_{i, j}, \\
n h_{i}=g_{T}^{A}\left(E_{i}\right) f^{A}\left(E_{i}\right) d E G,
\end{gathered}
$$

where $n h_{i}$ is the trapped electron density in the acceptor-like tail states and $f^{A}\left(E_{i}\right)$ is the function of the NDF for the acceptor-like trap states at the energy level $E_{i}$. The temperature dependence of $f^{A}\left(E_{i}\right)$ is especially important for the cause of the TQ. The trap states electron hopping photoconductivity at the energy level $E_{i}\left(\sigma_{\text {hop }}\left(E_{i}\right)\right)$ is shown as follows by Mott [6]:

$$
\sigma_{\text {hop }}\left(E_{i}\right)=\frac{q^{2}}{6 k_{B} T} R_{i, j}^{2} R_{h o, i}^{n} .
$$

The total trap states electron hopping photoconductivity $\left(\sigma_{\text {hop }}\right)$ is the summation of all the acceptor-like band tail energy levels:

$$
\sigma_{\text {hop }}=\frac{q^{2}}{6 k_{B} T} \sum_{i}\left(R_{i, j}^{2} R_{h o, i}^{n}\right)
$$

The band carrier' photoconductivity $\left(\sigma_{\text {ext }}\right)$ is calculated as follows with the electron density (n) in the CB and the hole density (p) in the VB:

$$
\sigma_{\text {ext }}=q\left(\mu_{n} n+\mu_{p} p\right)
$$

where $\mu_{n}$ and $\mu_{p}$ are mobility for the electrons in the CB and that for the holes in the VB, respectively. The trap states hopping and the band conduction occur simultaneously. So, the total photoconductivity $\sigma_{p h}$ is the 
summation of the trap states electron hopping photoconductivity and the band carriers' photoconductivity as follows:

$$
\sigma_{p h}=\sigma_{\text {hop }}+\sigma_{\text {ext }} .
$$

\subsection{Simulation parameters}

The parameters list of the present simulations is shown in Table $1[13,24,26]$. The device is p-type and n-type a$\mathrm{Si}: \mathrm{H}$. The length of the device is set to be sufficient length to examine the temperature dependence of the NDF; the longer the device size, the worse the convergence of the simulations at the low temperature.

Table 1 The parameters list of the present simulations.

\begin{tabular}{|c|c|c|}
\hline Parameter & Character (Unit) & Value \\
\hline Length of the device & $L(n m)$ & 150 \\
\hline Mobility-gap & $E_{g}(e V)$ & 1.72 \\
\hline Electron mobility & $\mu_{n}\left(\mathrm{~cm}^{2} \mathrm{~V}^{-1} \mathrm{sec}^{-1}\right)$ & 10 \\
\hline Hole mobility & $\mu_{p}\left(\mathrm{~cm}^{2} \mathrm{~V}^{-1} \mathrm{sec}^{-1}\right)$ & 0.3 \\
\hline Absorption coefficient & $\alpha\left(\mathrm{cm}^{-1}\right)$ & $1 \times 10^{4}$ \\
\hline Photon flux & $\Phi\left(\mathrm{cm}^{-2} \mathrm{sec}^{-1}\right)$ & $1.0 \times 10^{17}$ \\
\hline $\begin{array}{l}\text { Electron-hole pair generation rate per } \\
\text { unit volume (Eq. (28)) at the center of } \\
\text { the device }\end{array}$ & $\mathrm{G}\left(\mathrm{cm}^{-3} \mathrm{sec}^{-1}\right)$ & $1.74 \times 10^{21}$ \\
\hline Effective density of states of the $\mathrm{CB}$ & $N_{C}\left(\mathrm{~cm}^{-3}\right)$ & $1 \times 10^{19}$ \\
\hline Effective density of states of the VB. & $N_{V}\left(\mathrm{~cm}^{-3}\right)$ & $1 \times 10^{19}$ \\
\hline Ionized donor density & $N_{D}^{+}\left(\mathrm{cm}^{-3}\right)$ & $1 \times 10^{19}$ \\
\hline Ionized acceptor density & $N_{A}^{-}\left(\mathrm{cm}^{-3}\right)$ & $1 \times 10^{19}$ \\
\hline Thermal velocity & $v_{t h}\left(\mathrm{cmsec}^{-1}\right)$ & $1 \times 10^{7}$ \\
\hline The DOS of the tail states at $\mathrm{E}=E_{m}$ & $g_{0}\left(\mathrm{~cm}^{-3} \mathrm{eV}^{-1}\right)$ & $5.0 \times 10^{16}$ \\
\hline $\begin{array}{l}\text { Characteristic energies for the acceptor- } \\
\text { like tail states }\end{array}$ & $E_{A}(\mathrm{eV})$ & 0.053 \\
\hline $\begin{array}{l}\text { Characteristic energies for the donor- } \\
\text { like tail states }\end{array}$ & $E_{D}(e V)$ & 0.088 \\
\hline $\begin{array}{l}\text { Energy at which both tail states } \\
\text { coincide with the value of } g_{0}\end{array}$ & $E_{m}(e V)$ & 1.07 \\
\hline $\begin{array}{l}\text { Effective number density of states } \\
\text { associated with the acceptor-like deep } \\
\text { states }\end{array}$ & $N_{g}^{A}\left(\mathrm{~cm}^{-3}\right)$ & $1 \times 10^{15}$ \\
\hline
\end{tabular}


$\begin{array}{lll}\text { Effective number density of states } & N_{g}^{D}\left(\mathrm{~cm}^{-3}\right) & 1 \times 10^{15}\end{array}$

associated with the donor-like deep

states

Peak positions of the acceptor-like deep $\quad E_{g}^{A}(\mathrm{eV})$

states

Peak positions of the donor-like deep $\quad E_{g}^{D}(\mathrm{eV})$

states

Standard deviation in energy space of

$\sigma_{A}(e V)$

the acceptor-like deep states

Standard deviation in energy space of $\sigma_{D}(e V)$

the donor-like deep states

Electron capture cross-section of the $\sigma_{n}^{A}\left(\mathrm{~cm}^{2}\right)$ $1 \times 10^{-16}$ acceptor-like states

Hole capture cross-section of the $\sigma_{p}^{A}\left(c m^{2}\right)$ $1 \times 10^{-14}$ acceptor-like states

Electron capture cross-section of the $\sigma_{n}^{D}\left(c m^{2}\right)$ $1 \times 10^{-14}$ donor-like states

Hole capture cross-section of the donor$\sigma_{p}^{D}\left(c m^{2}\right)$ $1 \times 10^{-16}$ like states

$\begin{array}{lll}\text { Attempt to escape frequency } & v_{0}\left(\mathrm{sec}^{-1}\right) & 7 \times 10^{12} \\ \text { Localization radius of trap state } & \mathrm{a}(\mathrm{cm}) & 5.12 \times 10^{-8} \\ \text { The energy mesh interval in the } & \mathrm{dEG}(\mathrm{meV}) & 10 \\ \text { mobility-gap } & & \end{array}$

The temperature of this simulation is in the range of 150-400K. It has been shown that some of parameters have the temperature dependence and the energy dependence in previous studies. But we assume some of the parameters to be as follows and devise the parameter values.

- The mobility for the electrons in the $\mathrm{CB}$ and that for the holes in the VB are the constant values at $300 \mathrm{~K}$, respectively, without the temperature dependence. It has been shown that the mobility for band carriers decrease with decreasing temperature [33,34]; that has advantages in explaining the cause of the TQ. In order to clarify the effect of the temperature dependence of the NDF on the cause of the TQ, we deal with the mobility for band carriers without the temperature dependence.

- The values of the $N_{C}$ and the $N_{V}$ are set to be smaller than that of the tail states at the band edges. The reasons for that are as follows. The measured photoconductivities in Fig. 1 have the positive temperature dependence of the mobility for the band carriers [33,34]. But the simulations employ the mobilities without the temperature dependence as described above. That results in the large simulation value of $\sigma \_$ext at low temperature and the large deviation from the measured value of Fig. 1. In order to the discussion of the TQ observed around the temperature of 200K for p-type, the values of the $\sigma_{\text {ext }}$ are shifted to lower by setting the values of the $N_{C}$ and the $N_{V}$ to smaller values. 
- Capture cross-section for the carriers are the constant values at 300K (Table 1), without the energy dependence, without the temperature dependence and without the difference between tail states and DB.

- The donor and the acceptor are ionized completely in all temperature ranges.

\subsection{Simulation method}

The simulation scheme of the present study is the conventional drift-diffusion method; the numerical method is Scharfetter and Gummel's scheme with the iterative technique [35-39]. The device is divided into the meshes of $10 \mathrm{~nm}$ for calculating point. Imposing the nonequilibrium steady-state condition on each trap state, the NDF (the occupation probability of carriers for the trap states in the mobility-gap) are calculated self-consistently during the simulations, which treat the capture and emission processes rigorously. Figure 3 shows the flowchart of the driftdiffusion simulation in the present study [40]. The Poisson equation derives the electrostatic potential $\varphi$. The current continuity equation for the electrons derives the electron density $\mathrm{n}$ and that for the holes derives the hole density $\mathrm{p}$. The simulation derives $\varphi, n, p, n_{h}, p_{h}, n_{t}$, and $p_{t}$ self-consistently under the control of the distribution functions derived by employing the principle of the nonequilibrium steady-state condition, which is assumed that the capture and emission processes at the different energy $E$ in the mobility gap do not have a relationship with each other. Furthermore, employing Mott's hopping models, $\sigma_{\text {hop }}, \sigma_{\text {ext }}$ and $\sigma_{p h}$ are derived with the results of the self-consistent drift-diffusion simulation.

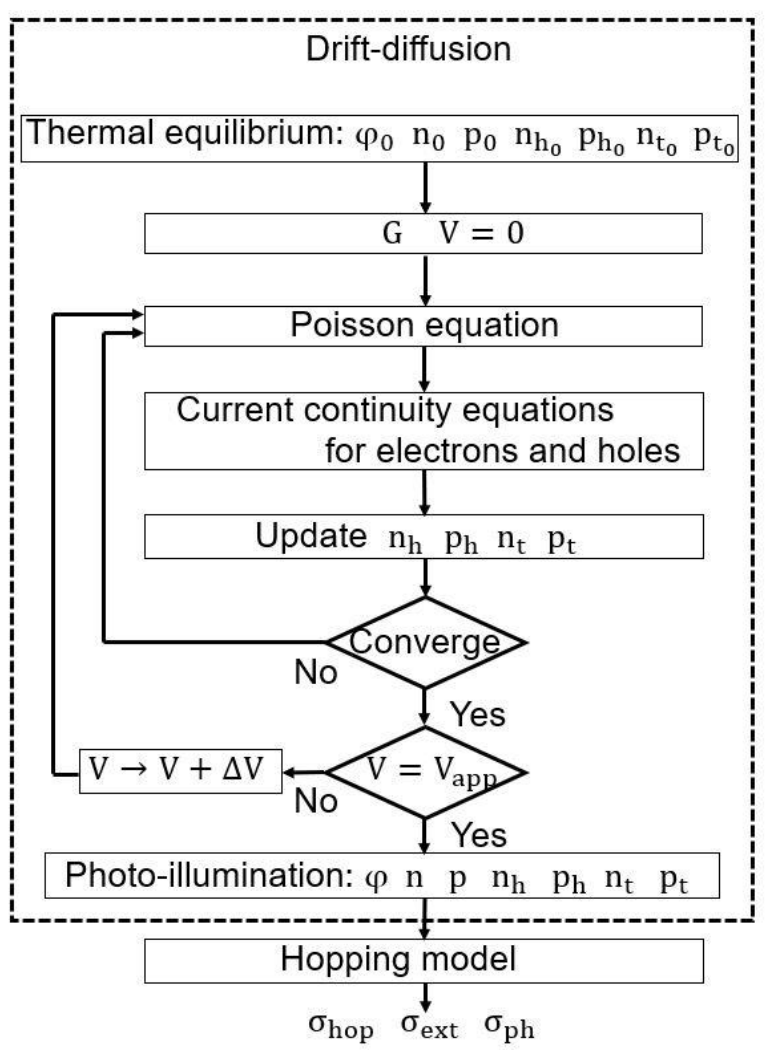


Fig. 3 The flowchart of the present drift-diffusion simulator. All calculations are carried out in terms of the distribution functions derived by employing the principle of the nonequilibrium steady-state condition for the acceptor-like and the donor-like states self-consistently. Vapp (bias voltage) is 0v in this study. Furthermore, employing Mott's hopping models, the photoconductivity is derived with the results of the self-consistent driftdiffusion simulation.

\section{Results and discussion}

\subsection{Temperature dependence of distribution functions}

\subsubsection{P-type}

Figure 4 shows the NDF for the acceptor-like trap states under the photo-illumination and zero bias voltage as a function of energy for p-type at the center of the device length at the temperature of $150 \mathrm{~K}, 200 \mathrm{~K}, 250 \mathrm{~K}, 300 \mathrm{~K}$, and $400 \mathrm{~K}$. On the other hand, Fig. 5 shows the EDF for the acceptor-like trap states as a function of energy for ptype at the center of the device length at the same temperatures. Both figures are shown on a semilogarithmic graph, with the abscissa denoting energy $(\mathrm{eV})$, and the ordinate denotes the electron occupation probability (logarithm scale). The slopes (occupation probability-to-energy gradient) of the NDF become gentler with increasing temperature, which are shown in the area surrounded by broken lines in Fig. 4; that is caused by the carriers' thermal excitation to the band. That is the same tendency for the slopes of the EDF shown in Fig. 5. The difference between the NDF and the EDF is that the NDF have stair shape structures: there are the energy regions to keep the values of the NDF constant against the energy.

Figures 6(a), (b) and (c) show the schematics of the capture and emission processes via the acceptor-like trap states located at energy $E$, which correspond to the energy levels [Region1], [Region2] and [Region3] in Fig. 4, respectively. The energy ranges of [Region1], [Region2] and [Region3] depend on temperature; those at 400K are shown in Fig. 4. Fig. 6(a) shows that when the energy level $E$ is far away from the CB and the VB, the emission process of the electrons and that of the holes $\left(b^{A}\right.$ and $\left.d^{A}\right)$ are much smaller than the capture process of the electrons and that of the holes $\left(a^{A}\right.$ and $\left.c^{A}\right)$. So, the transition rate of the electron capture process $a^{A}$ is mostly the same as that of the hole capture process $c^{A}\left(r_{a^{A}}=r_{c^{A}}\right)$; the electrons combine with the holes rapidly at the trap states. Under this condition, $f^{A}(E)$ have the constant values to become smaller with increasing temperature as shown in Fig. 4 [Region1]: the negative temperature dependence. The width of [Region1] becomes narrower with increasing temperature, because the transition rate of the hole emission process $d^{A}$ and that of the electron emission process $b^{A}$ become larger with increasing temperature. The constant values agree with the calculation values shown in Eq. (9): the values are determined by the density of the electrons in the $\mathrm{CB}$ and that of the holes in the VB. This means that the distribution functions are exactly calculated during simulations by employing the nonequilibrium steady-state condition for each trap state.

When the energy level $E$ gets closer to the edge of the $\mathrm{CB}$, the electron emission process $b^{A}$ comes into play as shown in Fig. 6(b). That makes the values of the NDF become small (Fig. 4 [Region2]). Focusing on the 
temperature dependence of the NDF at respective energy levels, the function values become smaller with increasing temperature: the negative temperature dependence. This is because that increasing temperature, the constant values of the NDF against the energy become smaller (Fig. 4 [Region1]), and the energy levels at which the NDF change from the constant to the decrease become lower: the width of [Region1] becomes narrower.

Further the energy level $E$ comes closer to the edge of the CB (Fig. 4 [Region3]), the electron capture and the electron emission processes $\left(a^{A}\right.$ and $\left.b^{A}\right)$ become dominant. In [Region3], according to the imposed the nonequilibrium steady-state condition, the small difference between $a^{A}$ and $b^{A}$ balances with the hole capture process $c^{A}$, as shown in Fig. 6(c). These capture and emission processes are rather similar to the equilibrium condition. But the condition of Fig. 6(c) is definitely not the equilibrium condition.

On the other hand, under the equilibrium condition, the values of the EDF near the CB for p-type become larger with increasing temperature as shown in Fig. 5: the positive temperature dependence. That is to say, the temperature dependence of the distribution functions for the trap states near the $\mathrm{CB}$ for $\mathrm{p}$-type under the nonequilibrium circumstances is the reverse of that under the equilibrium circumstances. That makes the difference in the temperature dependence of the trap states carriers' density and that of the hopping photoconductivity between both circumstances.

Now, we discuss the reason for the negative temperature dependence of the NDF near the CB for p-type. Employing the Shockley-Read-Hall recombination model for each energy level, the values of the NDF are determined by the density of the electrons in the CB and that of the holes in the VB. So, we discuss the temperature dependence of the density of the electrons in the $\mathrm{CB}$ and that of the holes in the VB as below. Considering the material temperature dependence, the carriers' generation rate per unit volume of photoillumination is constant without the temperature dependence. Figures 7(a) and 7(b) show the density of the electrons in the CB and that of the holes in the VB as a function of temperature for p-type and n-type, respectively. As for p-type, the density of the majority carrier holes produced by the photo generation and the thermal excitation in the VB increases exponentially with increasing temperature. On the other hand, the density of the minority carrier electrons in the $\mathrm{CB}$ is constant without the temperature dependence, because the density of the photo generation electrons without the temperature dependence is much larger than that of the thermal excitation electrons. In the Shockley-Read-Hall recombination model, the above-mentioned temperature dependence of band carriers' density makes the capture rate of holes for the trap states become larger with increasing temperature; in other words, the electron occupation probability for the trap states becomes smaller with increasing temperature. That explains that the values of the NDF become smaller with increasing temperature in the energy region near the $\mathrm{CB}$ for p-type. 


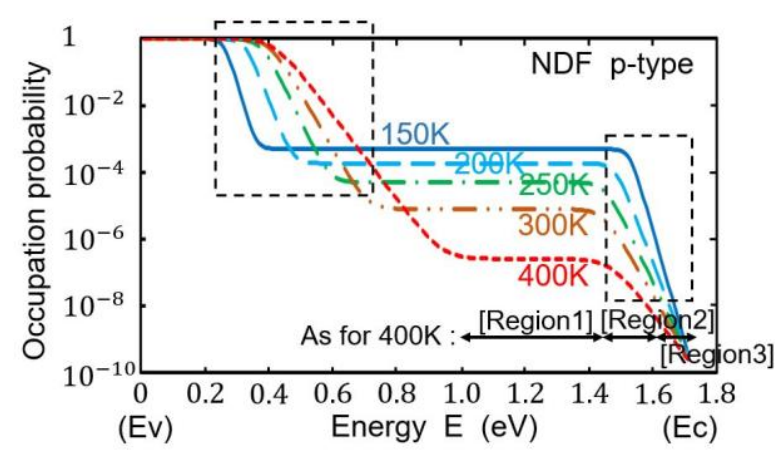

Fig. 4 The nonequilibrium distribution functions (NDF) for the acceptor-like trap states as a function of energy for p-type at the temperature of $150 \mathrm{~K}, 200 \mathrm{~K}, 250 \mathrm{~K}, 300 \mathrm{~K}$, and $400 \mathrm{~K}$. These functions have stair shape structures: there are the energy regions to keep the values of the NDF constant ([Region1]). In terms of the temperature dependence of these functions, the values of these functions become smaller with increasing temperature (the negative temperature dependence) in [Region1], [Region2] and [Region3].

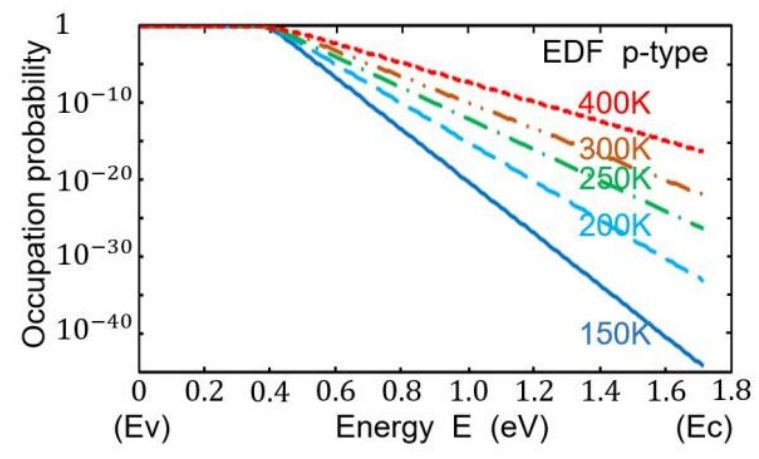

Fig. 5 The equilibrium distribution functions (EDF) for the acceptor-like trap states as a function of energy for ptype at the temperature of $150 \mathrm{~K}, 200 \mathrm{~K}, 250 \mathrm{~K}, 300 \mathrm{~K}$, and $400 \mathrm{~K}$.

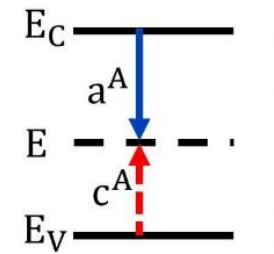

(a)

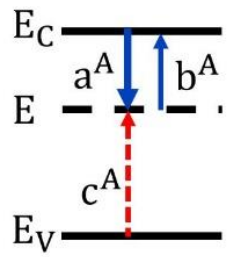

(b)

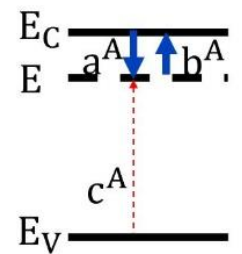

(c)

Fig. 6 The schematics of the capture and emission processes via the acceptor-like states located at energy $E$. (a) The energy level $E$ is far away from the conduction band and the valence band, the capture process of the electrons and that of the holes $\left(a^{A}\right.$ and $c^{A}$ ) are dominant. (b) The energy level $E$ gets closer to the edge of the conduction band, the electron emission process $b^{A}$ comes into play. (c) The energy level $E$ is remarkably close to the edge of the conduction band, the electron capture and the electron emission processes $\left(a^{A}\right.$ and $\left.b^{A}\right)$ become dominant, and the 
small difference between $a^{A}$ and $b^{A}$ balances with the hole capture process $c^{A}$. (a), (b) and (c) correspond to the energy level [Region1], [Region2] and [Region3] in Fig. 4, respectively.
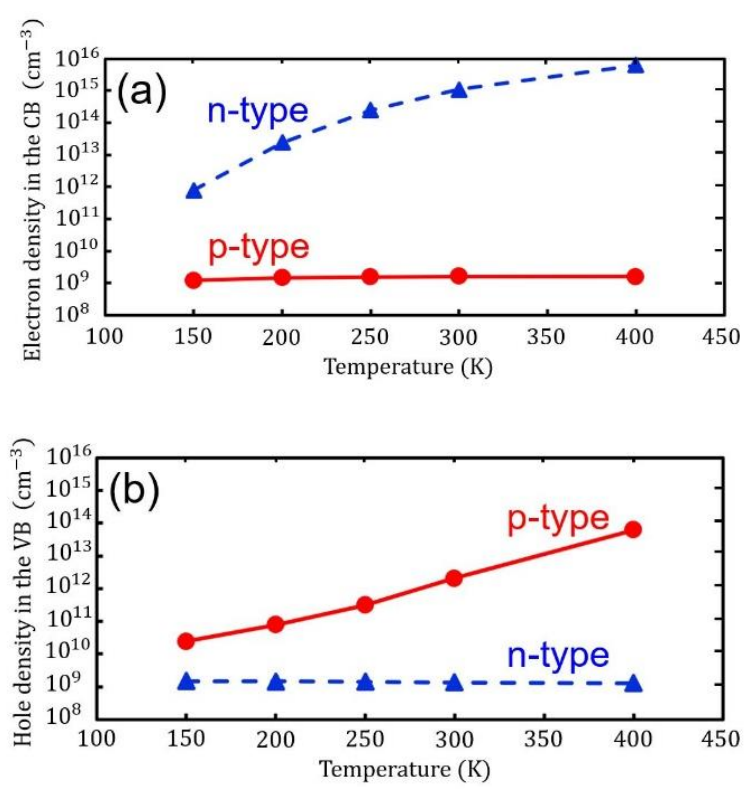

Fig. 7 (a) The electron density in the conduction band as a function of temperature for p-type and n-type. (b) The hole density in the valence band as a function of temperature for p-type and n-type. The densities of the majority carriers produced by the photo generation and the thermal excitation in the bands increase exponentially with increasing temperature. On the other hand, the densities of the minority carriers in the bands are constant without the temperature dependence, because the densities of the minority carriers produced by the photo generation are much larger than those produced by the thermal excitation.

\subsubsection{N-type}

Figures 8(a) and 8(b) show the same NDF for the acceptor-like trap states as a function of energy for n-type at the center of the device length at the temperature of $150 \mathrm{~K}, 200 \mathrm{~K}, 250 \mathrm{~K}, 300 \mathrm{~K}$, and $400 \mathrm{~K}$. Figure $8(\mathrm{a})$ is shown on linear graph; the inset is the enlarged figure in the area surrounded by the broken line. On the other hand, Fig. 8(b) is shown on a semilogarithmic graph.

The common characteristics between the NDF for n-type and that for p-type (Fig. 4) are as follows.

- Both NDF have stair shape structures: there are the energy regions to keep the values of the NDF constant. 
- The widths of the energy regions to keep the values of the NDF constant become narrower with increasing temperature; that is because that the transition rate of the hole emission process $d^{A}$ and that of the electron emission process $b^{A}$ become larger with increasing temperature.

- The slopes (occupation probability-to-energy gradient) of the NDF become gentler with increasing temperature; that is caused by the carriers' thermal excitation to the band.

On the other hand, the different characteristics between the NDF for n-type and that for p-type are as follows.

- The constant values of the NDF against the energy for n-type become larger with increasing temperature (the positive temperature dependence) as shown in the vicinity of the energy area surrounded by the broken line in Fig. $8(a)$, which is the reverse of the temperature characteristic for p-type.

- In the vicinity of the $\mathrm{CB}$, the values of the NDF at respective energy level become larger with increasing temperature just like the EDF (the positive temperature dependence) as shown in the area surrounded by the broken line in Fig. 8(b). So, the temperature dependence of the NDF near the CB for n-type is the reverse of that for p-type.

We show the difference of the temperature characteristic between the NDF for n-type and that for p-type by using the explanatory figures Fig. 9(a) for n-type and Fig. 9(b) for p-type. The solid lines show the NDF for the lower temperature, and the broken lines show the NDF for the higher temperature. The NDF are exactly calculated during simulations by employing the nonequilibrium steady-state condition for each trap state. As already mentioned earlier, when the energy level $E$ is far away from the $\mathrm{CB}$ and the VB, the transition rate of the electron capture process $a^{A}$ is mostly the same as that of the hole capture process $c^{A}\left(r_{a^{A}}=r_{c^{A}}\right)$, and the NDF have the constant values against the energy. As for the temperature dependence of the constant values of the NDF against the energy, the values become larger for n-type, and those become smaller for p-type with increasing temperature because of the temperature dependence of the densities of the band carriers (Fig. 7(a) and (b)).

Concerning the values of the NDF near the $\mathrm{CB}$, the values are affected by the constant values of the NDF against the energy, the energy levels at which the NDF change from the constant to the decrease, and the slope of the NDF. For n-type, the constant values of the NDF of the higher temperature are larger than those of the lower temperature, and the slopes of the NDF of the higher temperature are the gentler than those of the lower temperature. That makes the values of the NDF at respective energy level become larger with increasing temperature near the CB for n-type.

The dope densities and the light intensity influence the temperature characteristic of the NDF. In this study, the densities of ionized donor and acceptor are typical values, and photon flux is about AM1.5 as shown in Table 1.

Those differences between both types as shown in Fig. 9 are the cause of the TQ that is observed for p-type, but not observed for n-type in a low temperature around 200K. That is explained in detail in the next section.

For reference, as for the hole distribution functions, the values of the nonequilibrium hole distribution functions for the donor-like trap states in the mobility-gap $\left(1-f^{D}(E)\right)$ decrease with increasing temperature (the negative temperature dependence) in the energy region near the VB for n-type. 

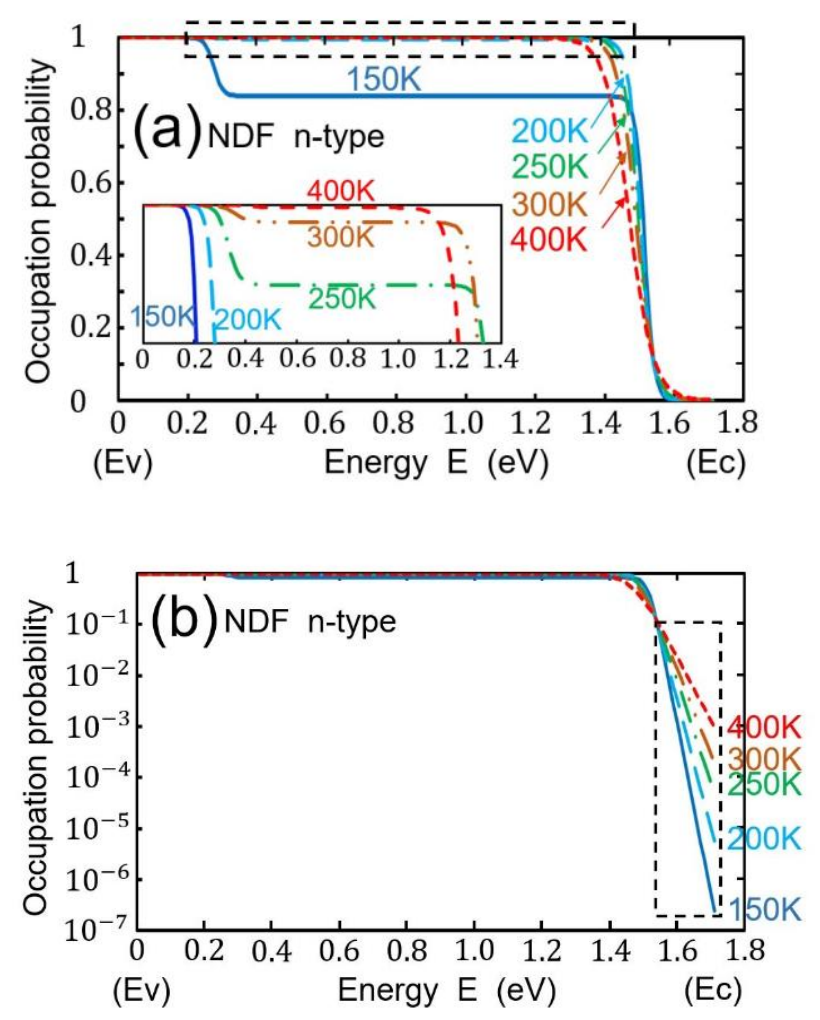

Fig. 8 The nonequilibrium distribution functions for the acceptor-like trap states as a function of energy for n-type at the temperature of $150 \mathrm{~K}, 200 \mathrm{~K}, 250 \mathrm{~K}, 300 \mathrm{~K}$, and $400 \mathrm{~K}$. (a) That is shown on linear graph; the inset is the enlarged figure in the area surrounded by the broken line. These functions have stair shape structures: there are the energy regions to keep the values of the NDF constant. The constant values become larger with increasing temperature (the positive temperature dependence). (b) That is shown on a semilogarithmic graph. In the vicinity of the $\mathrm{CB}$, the values of the NDF at respective energy level become larger with increasing temperature just like the $\mathrm{EDF}$ (the positive temperature dependence) as shown in the area surrounded by the broken line.

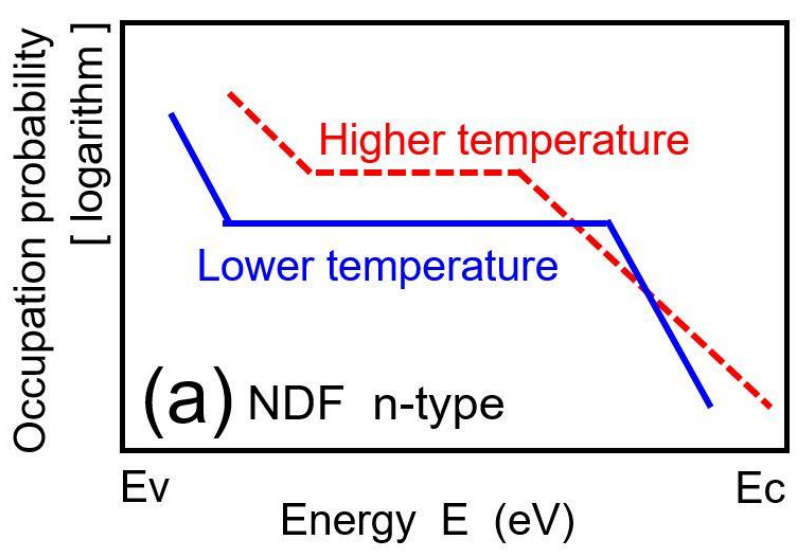




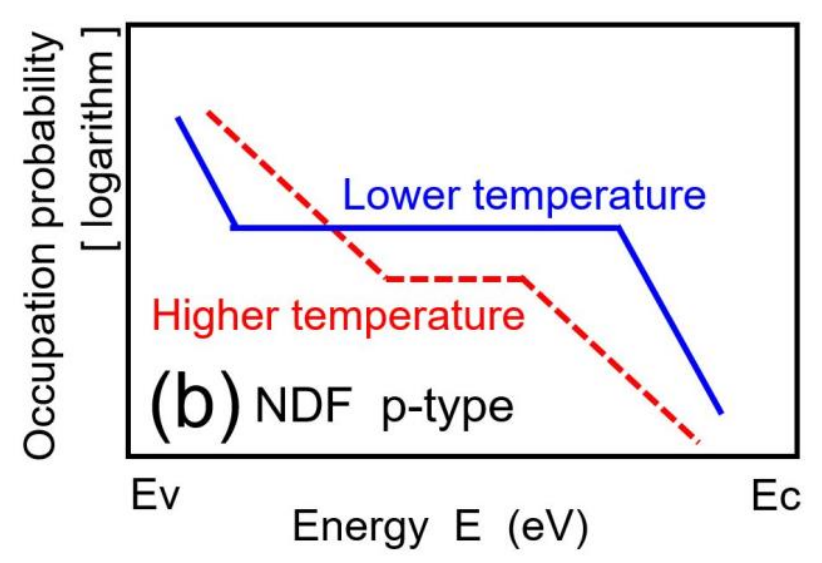

Fig. 9 The explanatory figures to show the difference of the temperature characteristic between the NDF for n-type and that for p-type. (a) The NDF for n-type. (b) The NDF for p-type. The solid lines show the NDF for the lower temperature, and the broken lines show the NDF for the higher temperature.

\subsection{Temperature dependence of photoconductivity}

We consider that the electron hopping occurs between two energy levels in conduction band tail states. Figure 10(a) shows the summations of the hopping rates per unit volume $R_{h o, i}^{n}$ (Eq. (35)) for the trapped electrons in the acceptor-like band tail for p-type, which are the hopping from the initial state at the energy level $E_{i}$ to a lot of destination states at the energy level $E_{j}$, as a function of the initial energy level $E_{i}$ at the temperature of $150 \mathrm{~K}$, $200 \mathrm{~K}, 250 \mathrm{~K}, 300 \mathrm{~K}$, and $400 \mathrm{~K}$. Figure 10 (b) is the enlarged figure of Fig. 10(a) near the CB. The calculation energy mesh interval in the mobility-gap is $10 \mathrm{meV}$. It is found that $R_{h o, i}^{n}$ are changed exponentially against the initial energy level $E_{i}$, and $R_{h o, i}^{n}$ near the CB is dominant, which is caused by the DOS of the tail states that increases exponentially against the energy level (Eq. (15)). Focusing on the temperature dependence of $R_{h o, i}^{n}$ near the CB, the values of $R_{h o, i}^{n}$ become smaller with increasing temperature because of the negative temperature dependence of the NDF near the CB for p-type (Fig. 4). So, the total hopping rates per unit volume $\sum_{i} R_{h o, i}^{n}$, which are the summation of $R_{h o, i}^{n}$ at all the initial energy level $E_{i}\left(E_{V} \leq E_{i} \leq E_{C}\right)$, decrease with increasing temperature; that is the negative temperature dependence.

Figure 11(a) shows the summations of the hopping rates per unit volume $R_{h o, i}^{n}$ for n-type as a function of the initial energy level $E_{i}$ at the temperature of $150 \mathrm{~K}, 200 \mathrm{~K}, 250 \mathrm{~K}, 300 \mathrm{~K}$, and $400 \mathrm{~K}$. Figure $11(\mathrm{~b})$ is the enlarged figure of Fig. 11(a) near the CB. It is also found that $R_{h o, i}^{n}$ are changed exponentially against the initial energy level $E_{i}$, and $R_{h o, i}^{n}$ near the $\mathrm{CB}$ is dominant. As for the temperature dependence of $R_{h o, i}^{n}$ near the $\mathrm{CB}$, the values of $R_{h o, i}^{n}$ increase with increasing temperature because of the positive temperature dependence of the NDF near the CB for n-type (Fig. 8(b)). So, the total hopping rates per unit volume $\sum_{i} R_{h o, i}^{n}$ increase with increasing temperature; that is the positive temperature dependence. That is to say, the temperature dependence of $\sum_{i} R_{h o, i}^{n}$ for n-type is the reverse of that for p-type.

Figure 12(a) shows the electron hopping photoconductivity $\sigma_{\text {hop }}$ calculated from Eq. (38) as a function of temperature for p-type and n-type, respectively. The photoconductivity depends on the illumination intensity; the normalized photoconductivity $\sigma_{\text {hop }} / q G$ is shown. $G$ is described in the Table $1 . \sigma_{\text {hop }}$ for p-type decreases with 
increasing temperature (the negative temperature dependence) because of the negative temperature dependence of the NDF near the CB. But $\sigma_{\text {hop }}$ for n-type increases with increasing temperature (the positive temperature dependence) because of the positive temperature dependence of the NDF. Figure 12(b) shows the band carriers' photoconductivity $\sigma_{\text {ext }}$ as a function of temperature for p-type and n-type, respectively. $\sigma_{\text {ext }}$ consists of the photoconductivity for the electrons in the $\mathrm{CB}$ and that for the holes in the VB, which is calculated from Eq. (39). $\sigma_{\text {ext }}$ for both types increase monotonically because of the thermal excitation of the band carriers. Figure 12(c) shows the total photoconductivity $\sigma_{p h}$, which is the summation of $\sigma_{\text {hop }}$ and $\sigma_{\text {ext }}$, calculated from Eq. (40) as a function of temperature for p-type and n-type, respectively. As for p-type, the minimum of $\sigma_{p h}$ is shown at 200K. These figures show that the electron hopping photoconductivity having the negative temperature dependence is larger than the band carriers' photoconductivity having the positive temperature dependence less than $200 \mathrm{~K}$. Meanwhile $200 \mathrm{~K}$ or more, the band carriers' photoconductivity is larger than the electron hopping photoconductivity. For these reasons, the total photoconductivity $\sigma_{p h}$ changes from the decrease to the increase with increasing temperature around $200 \mathrm{~K}$. According to these results, the TQ is explained by the negative temperature dependence of the NDF near the CB for p-type. As for n-type, $\sigma_{p h}$ increases monotonically and the minimum value of $\sigma_{p h}$ is not shown in Fig. 12(c), which is because that the NDF for n-type do not have the negative temperature dependence but have the positive temperature dependence.

It is considered that the TQ observed around the temperature of 350K for n-type in Fig. 1 is explained not by the hopping model with the positive temperature dependence of the NDF, but by the Tran's assumption described in the introduction.
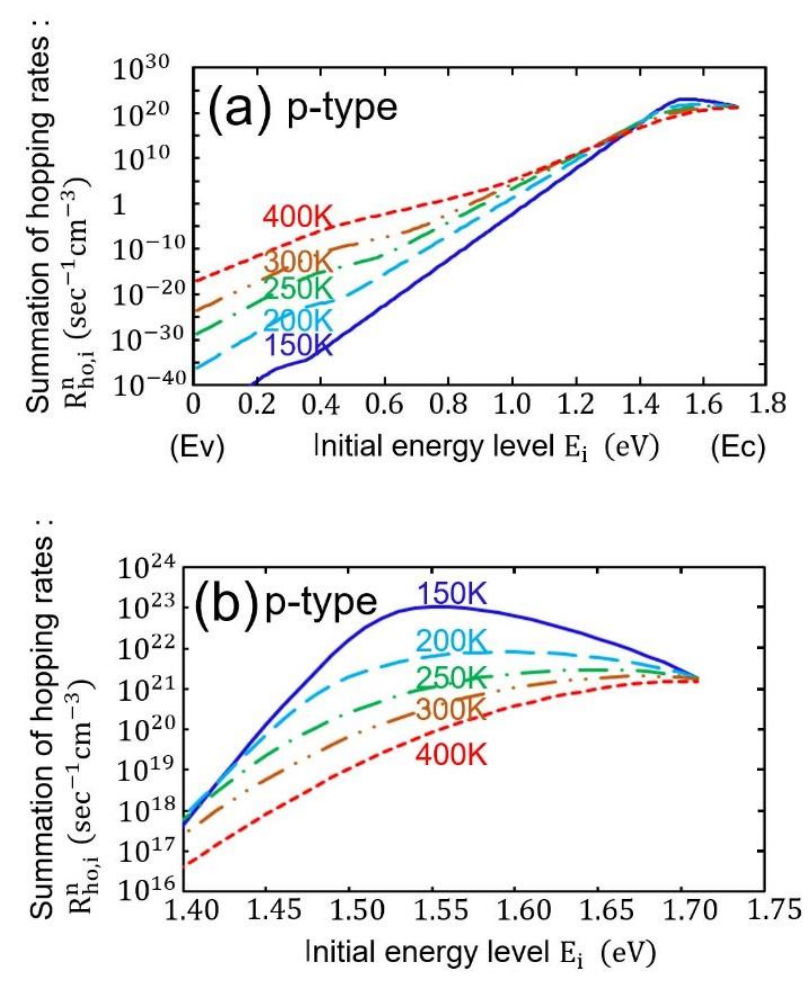
Fig. 10 The summations of the hopping rates per unit volume $R_{h o, i}^{n}$ (Eq. (35)) for the trapped electrons in the acceptor-like band tail for p-type as a function of the initial energy level $E_{i}$ at the temperature of $150 \mathrm{~K}, 200 \mathrm{~K}$, $250 \mathrm{~K}, 300 \mathrm{~K}$, and $400 \mathrm{~K}$. (a) That is shown in the whole energy level of the mobility-gap. It is found that $R_{h o, i}^{n}$ near the conduction band is dominant. (b) That is shown in the vicinity of the conduction band; in terms of the temperature dependence of $R_{h o, i}^{n}$, the values of $R_{h o, i}^{n}$ become smaller with increasing temperature (the negative temperature dependence).
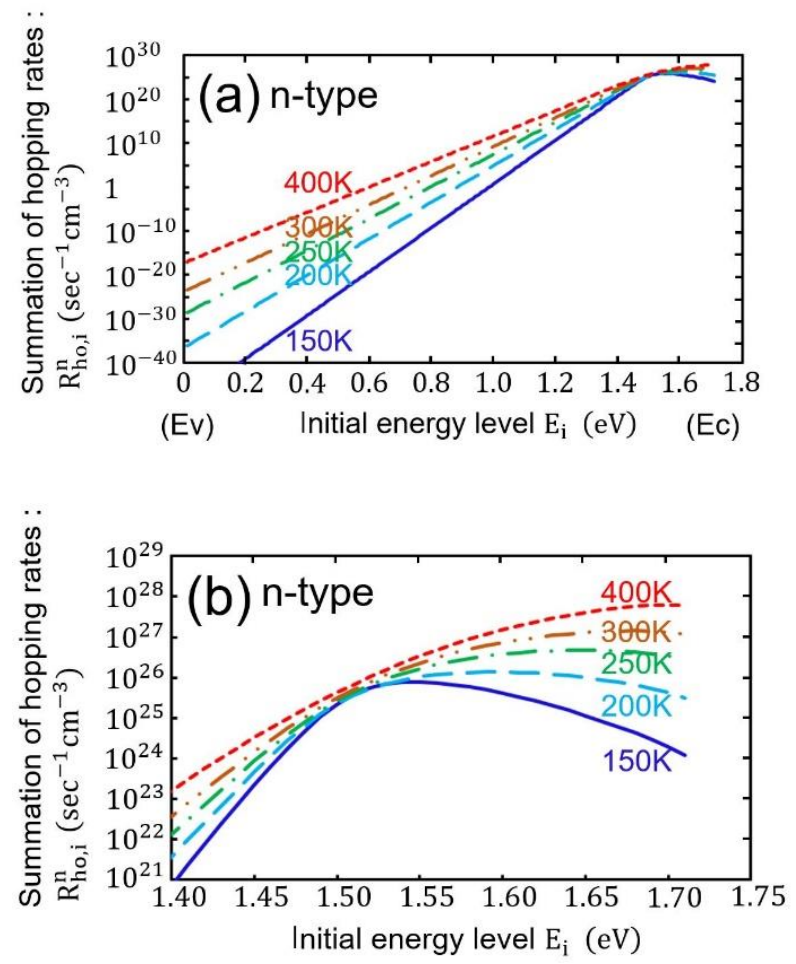

Fig. 11 The summations of the hopping rates per unit volume $R_{h o, i}^{n}$ (Eq. (35)) for the trapped electrons in the acceptor-like band tail for n-type as a function of the initial energy level $E_{i}$ at the temperature of $150 \mathrm{~K}, 200 \mathrm{~K}$, $250 \mathrm{~K}, 300 \mathrm{~K}$, and $400 \mathrm{~K}$. (a) That is shown in the whole energy level of the mobility-gap. It is found that $R_{h o, i}^{n}$ near the conduction band is dominant. (b) That is shown in the vicinity of the conduction band; in terms of the temperature dependence of $R_{h o, i}^{n}$, the values of $R_{h o, i}^{n}$ become larger with increasing temperature (the positive temperature dependence). The temperature dependence of $R_{h o, i}^{n}$ for n-type is the reverse of that for $\mathrm{p}$-type. 


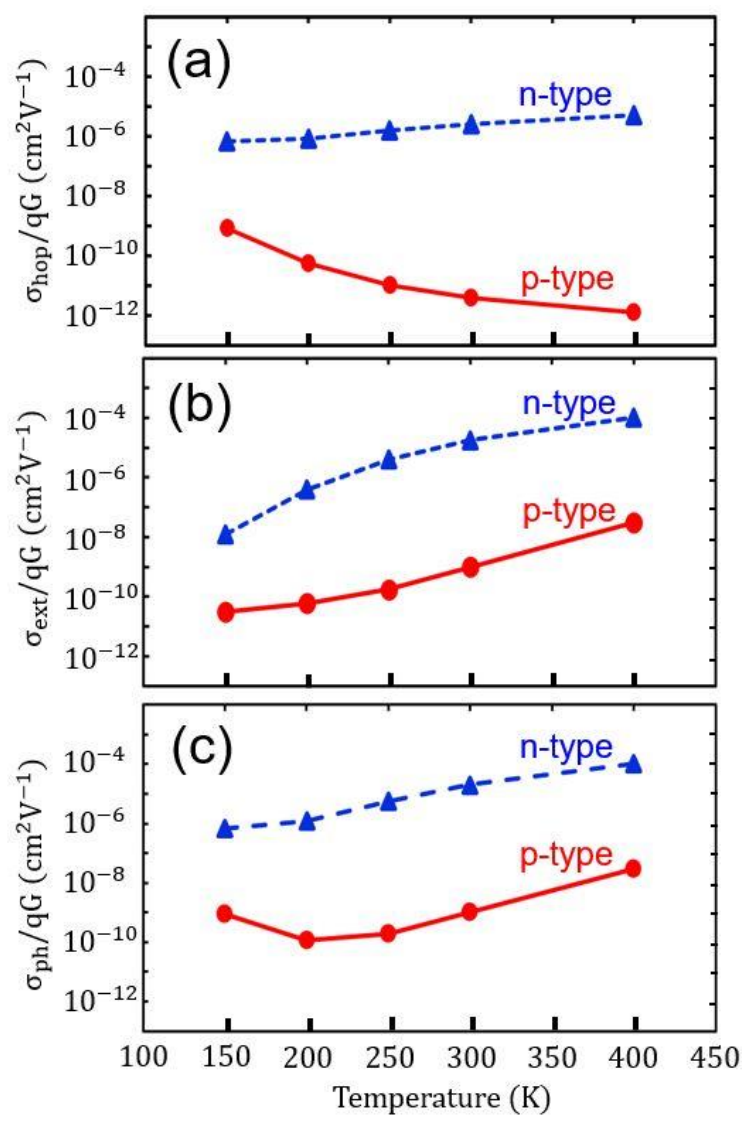

Fig. 12 The calculation results of the photoconductivity as a function of temperature for p-type and n-type. The photoconductivity is normalized by illumination intensity. (a) The electron hopping photoconductivity $\sigma_{\text {hop }}$ calculated from Eq. (38). (b) The band carriers' photoconductivity $\sigma_{\text {ext }}$, which consists of the photoconductivity for the electrons in the conduction band and that for the holes in the valence band, calculated from Eq. (39). (c) The total photoconductivity $\sigma_{p h}$, which is the summation of $\sigma_{\text {hop }}$ and $\sigma_{\text {ext }}$, calculated from Eq. (40). The thermal quenching is shown in the low temperature around $200 \mathrm{~K}$ for p-type.

\section{Conclusions}

The nonequilibrium distribution functions (NDF) for the trap states in the mobility-gap under photo illumination and zero bias voltage is derived by the constructed self-consistent drift-diffusion simulator consisted of the Poisson equation and current continuity equations for a-Si:H. As for the temperature dependence of the NDF, we find that the values of the NDF decrease with increasing temperature (the negative temperature dependence) in the energy region near the $\mathrm{CB}$ for p-type, which is the reverse of the temperature dependence of the equilibrium distribution functions (EDF) for the trap states in the mobility-gap, for the first time ever. As for n-type, the values of the NDF increase with increasing temperature (the positive temperature dependence) in the energy region near the $\mathrm{CB}$, which is the same as the EDF. The temperature dependence of the NDF is caused by that of the densities of the carriers produced by the photo generation and the thermal excitation in the bands and the balance of capture and emission rates between the majority carriers and the minority carriers in the bands. 
Furthermore, we show that the new physical characteristic is applicable to the explanation of the temperature characteristic of the photoconductivity caused by the electron hopping in the conduction band tail for a-Si:H. Employing Mott's hopping models, the electron hopping photoconductivity is derived by the summation of hopping rates for all hopping paths, which is related to the product of the DOS of the trap states in the mobility-gap and the NDF. The DOS of the tail states near the CB is large, which makes the hopping rates near the CB become dominant. As for the temperature dependence of the NDF near the CB for p-type, the values of the NDF decrease with increasing temperature (the negative temperature dependence). These make the electron hopping photoconductivity decrease with increasing temperature for p-type. In the lower temperature less than $200 \mathrm{~K}$, the electron hopping photoconductivity having the negative temperature dependence is larger than the band carriers' photoconductivity having the positive temperature dependence. Meanwhile 200K or more, the band carriers' photoconductivity gets ahead of the electron hopping photoconductivity. So, the temperature dependence of the total photoconductivity that is the summation of the electron hopping photoconductivity and the band carriers' photoconductivity changes from the decrease to the increase with increasing temperature around 200K.

Thus, we show that the TQ observed in a low temperature around 200K for p-type a-Si:H can be explained by the electron hopping model with the p-type NDF having the negative temperature dependence, for the first time ever.

\section{Acknowledgments}

The author would like to thank Prof. Nobuyuki Sano from the University of Tsukuba for the discussion during this study.

\section{References}

1. Simmons, J. G., Taylor, G. W.: Nonequilibrium steady-state statistics and associated effects for insulators and semiconductors containing an arbitrary distribution of traps. Phys. Rev. B 4(2), 502-511 (1971)

2. Taylor, G. W.: Comments on 'Surface-state density by photovoltage measurements-II'. J. Phys. D 5, 52-54 (1972)

3. Taylor, G. W, Simmons, J. G.: Basic equations for statistics, recombination processes, and photoconductivity in amorphous insulators and semiconductors. J. Noncryst. Solids 8-10, 940-946 (1972)

4. Simmons, J. G., Taylor, G. W.: The theory of photoconductivity in defect insulators containing discrete trap levels. J. Phys. C 8, 3353-3359 (1975)

5. Shockley, W., Read, W. T.: Statistics of the recombinations of holes and electrons. Phys. Rev. 87(5), 835-842 (1952)

6. Mott, N.F., Davis, E. A.: Electronic processes in non-crystalline materials. Clarendon Press, Oxford (1979)

7. Marshall, J. M.: Computer-assisted study of carrier thermalization by hopping in disordered semiconductors. J. materials science: materials in electronics 14, 611-614 (2003) 
8. Marshall, J. M.: Analytical procedures for the modelling of hopping transport in disordered semiconductors. Phil. Mag. Lett. 80(10), 691-701 (2000)

9. Godet, C.: Hopping model for charge transport in amorphous carbon. Phil. Mag. B 81(2). 205-222 (2001)

10 Fritzsche, H., Tran, M. Q., Yoon, B. -G., Chi, D. -Z.: The sign of photocarriers and thermal quenching of photoconductivity in a-SiH. J. Non-Cryst. Solids 137\&138, 467-470 (1991)

11. Fritzsche, H., Yoon, B. -G., Chi, D. -Z., Tran, M.Q.: Tran, Some observations on the photoconductivity of amorphous semiconductors. J. Non-Cryst. Solids 141, 123-132 (1992)

12. Tran, M.Q.: On thermal quenching of the photoconductivity in hydrogenated amorphous silicon. Phil. Mag. B 72(1), 35-66 (1995)

13. Merazga, A., Tobbeche, S., Main, C., Al-Shahrani, A., Reynolds, S.: Numerical simulation of the steady state photoconductivity in hydrogenated amorphous silicon including localized state electron hopping. J. Phys.:

Condens. Matter 18, 3721-3734 (2006)

14. Vanier, P. E., Delahoy, A. E., Griffith, R. W.: New features of the temperature dependence of photoconductivity in plasma-deposited hydrogenated amorphous silicon alloys. J. Appl. Phys. 52, 5235-5242 (1981)

15. Persans, P. D.: Dual-beam photoconductivity modulation spectroscopy in a-Si: H. Philos. Mag. B 46, 435-471 (1982)

16. Vanier, P. E., Griffith, R. W.: Infrared quenching of photoconductivity and the study of gap states in hydrogenated amorphous silicon alloys. J. Appl. Phys. 53, 3098-3102 (1982)

17. Dersch, H., Schweitzer, L.: Spin-dependent hole diffusion in a-Si: H. Philos. Mag. B 50, 397-404 (1984)

18. Carius, R., Fuhs, W., Hoheisel, M.: Infrared quenching of photoluminescence and photoconductivity in aSi:H. J. Non-Cryst. Solids 66, 151-156 (1984)

19. Fuhs, W.: Recombination of trapped carriers in a-Si:H probed by subbandgap excitation. J. Non-Cryst. Solids 77\&78, 593-602 (1985)

20. McMahon, T. J., Xi, J. P.: Photoconductivity and light-induced change in a-Si:H. Phys. Rev. B 34, 2475-2481 (1986)

21. Vaillant, F., Jousse, D., Bruyere, J.-C.: Recombination at dangling bonds and band tails: Temperature dependence of photoconductivity in hydrogenated amorphous silicon. Philos. Mag. B 57, 649-661 (1988)

22. Hack, M., Shur, M.: Theoretical modeling of amorphous silicon-based alloy p-i-n solar cells. J. Appl. Phys. 54, 5858-5863 (1983)

23. Hack, M., Guha, S., Shur, M.: Photoconductivity and recombination in amorphous silicon alloys. Phys. Rev. B 30 (12), 6991-6999 (1984)

24. Hack, M., Shur, M.: Physics of amorphous silicon alloy p-i-n solar cells. J. Appl. Phys. 58, 997-1020 (1985)

25. Hack, M., Shur, M.: Analysis of light-induced degradation in amorphous silicon alloy p-i-n solar cells. J. Appl. Phys. 58, 1656-1661 (1985)

26. Fantoni, A., Vieria, H., Martins R.: Simulation of hydrogenated amorphous and microcrystalline silicon optoelectronic devices. Math. Comput. Simul. 49, 381-401 (1999) 
27. Fantoni, A., Vieria, H., Martins, R.: Influence of the intrinsic layer characteristics on a-Si:H p-i-n solar cell performance analysed by means of a computer simulation. Sol. Energy Mater. Sol. Cell 73, 151-162 (2002)

28. Walker, P.H., Uno, S., Mizuta, H.: Simulation study of the dependence of submicron polysilicon thin-film transistor output characteristics on grain boundary position. Jpn. J. Appl. Phys. 44(12), 8322-8328 (2005).

29. Turner,G.B., Schwartz, R.J., Park, J.W., Gary, J.L.: Recombination in thin film Si:H p-i-n solar cells. J. NonCryst. Solids 97 \& 98, 1307-1310 (1987)

30. Nawaz, M.: Computer analysis of thin-film amorphous silicon heterojunction solar cells, J. Phys. D 44, 145105 (13pp) (2011)

31. Tiedje, T., Rose, A.: A physical interpretation of dispersive transport in disordered semiconductors. Solid State Commun. 37 (1), 49-52 (1980.

32. Tiedje, T.: Band tail recombination limit to the output voltage of amorphous silicon solar cells. Appl. Phys. Lett. 40, 627-629 (1982)

33. Le Comber, P. G., Spear, W. E.: Electronic Transport in Amorphous Silicon Films. Phys. Rev. Lett. 25, 509$511(1970)$

34 Tiedje, T., Cebulka, J. M., Morel, D. L., Abeles, B.: Evidence for Exponential Band Tails in Amorphous Silicon Hydride. Phys. Rev. Lett. 46, 1425-1428 (1981)

35. Schropp, R.E.I., Zeman, M.: Amorphous and Microcrystalline Silicon Solar Cells. Kluwer, Norwell (1998)

36. Nelson, J.: The Physics of Solar Cells. Imperial College Press, London (2003)

37. Wurfel, P.: Physics of Solar Cells, From Basic Principles to Advanced Concepts. WILEY-VCH Verlag GmbH \& Co.KGaA (2009)

38. Selberherr, S.: Analysis and Simulation of Semiconductor Devices. Springer-Verlag, Wien, New York (1984)

39. Scharfetter, H.K., Gummel, H.K.: Large-signal analysis of a silicon read diode oscillator. IEEE Trans. Electron Dev. ED-16 (1), 64-77 (1969)

40. Suzuki, A., Yoshida, K., Sano, N.: Self-consistent device simulation of a-Si p-i-n solar cells and energy resolution analyses of capture and emission processes. J. Comput. Electron. 15 (4), 1554-1562 (2016) 
Figures

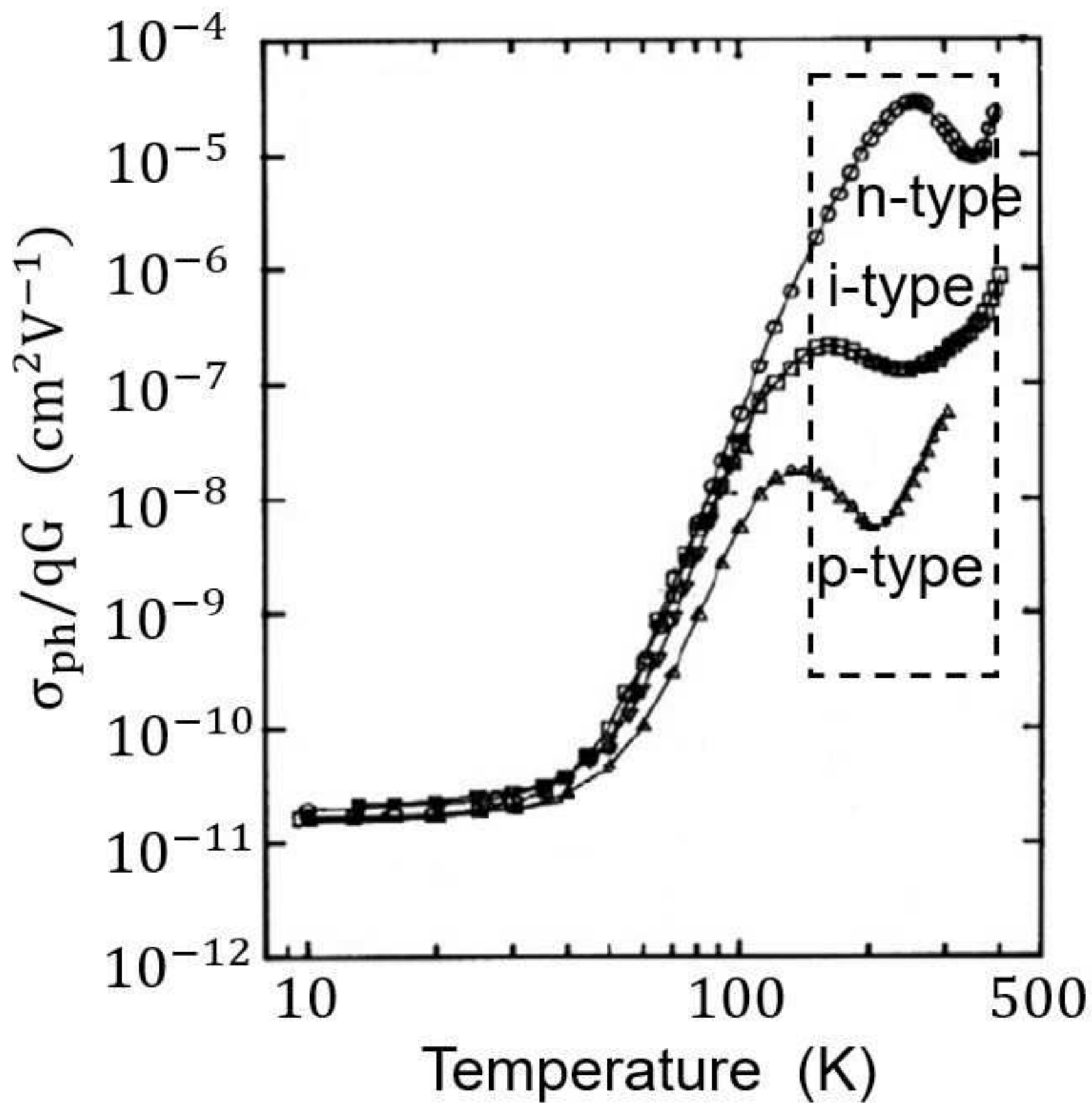

Figure 1

The typical measured photoconductivity in a-Si:H for p-type, i-type and n-type as a function of temperature $[10,11]$. In the area surrounded by broken line, the photoconductivity turns over from the increase to the decrease with increasing temperature. Further, the photoconductivity turns over from the decrease to the increase with more increasing temperature. This temperature characteristic is called the thermal quenching (TQ). 


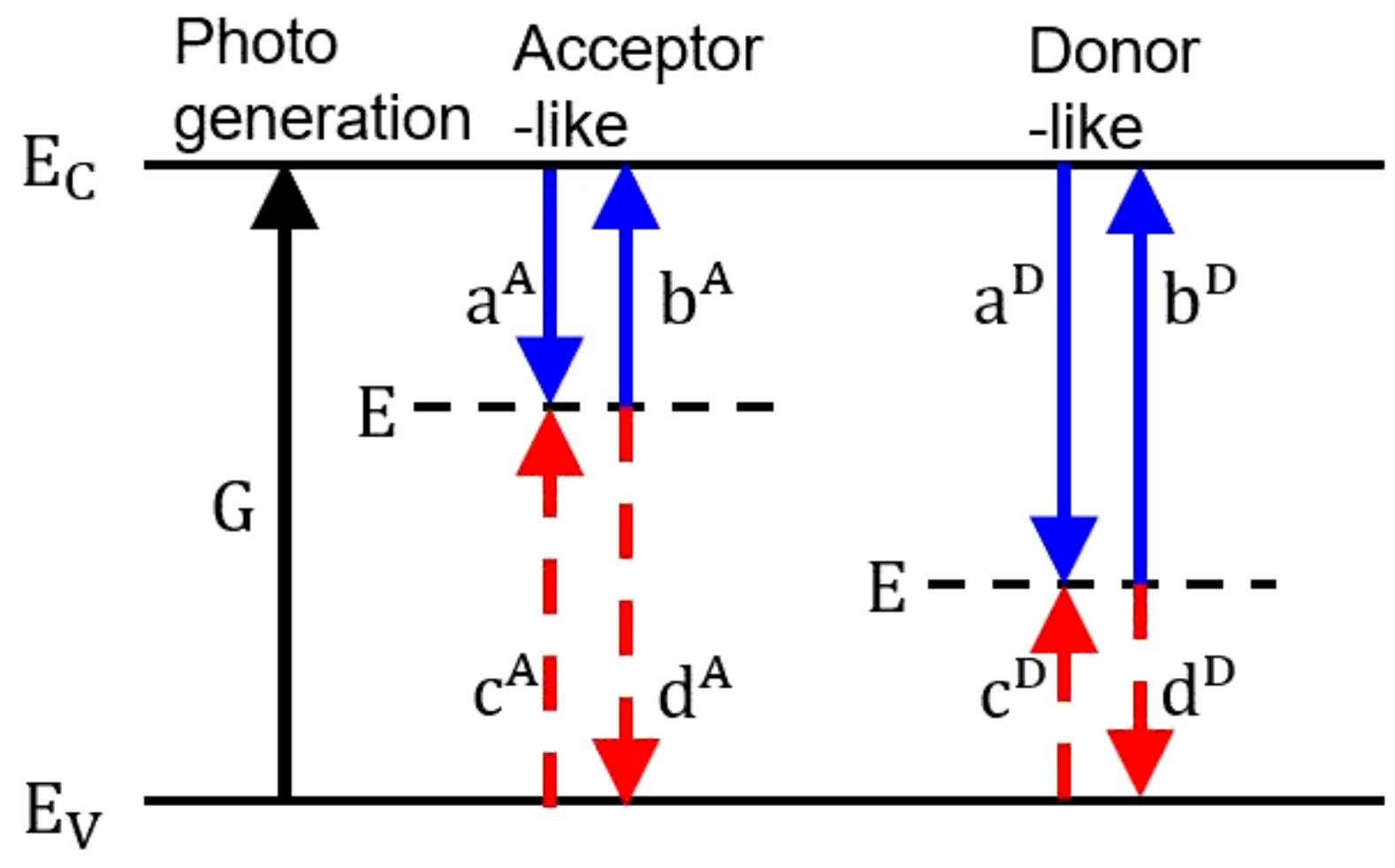

Figure 2

Two capture and two emission processes of the electrons and the holes via the acceptor-like trap states located at energy $E\left(a^{\wedge} A, b^{\wedge} A, c^{\wedge} A\right.$, and $\left.d^{\wedge} A\right)$ and those via the donor-like trap states located at energy $E$ $\left(a^{\wedge} D, b^{\wedge} D, c^{\wedge} D\right.$, and $\left.d^{\wedge} D\right)$ under photo-illumination. The solid line arrows and the broken line arrows indicate the transition directions of the electrons and the holes, respectively. The electron photo generation process $\mathrm{G}$ is also shown. 


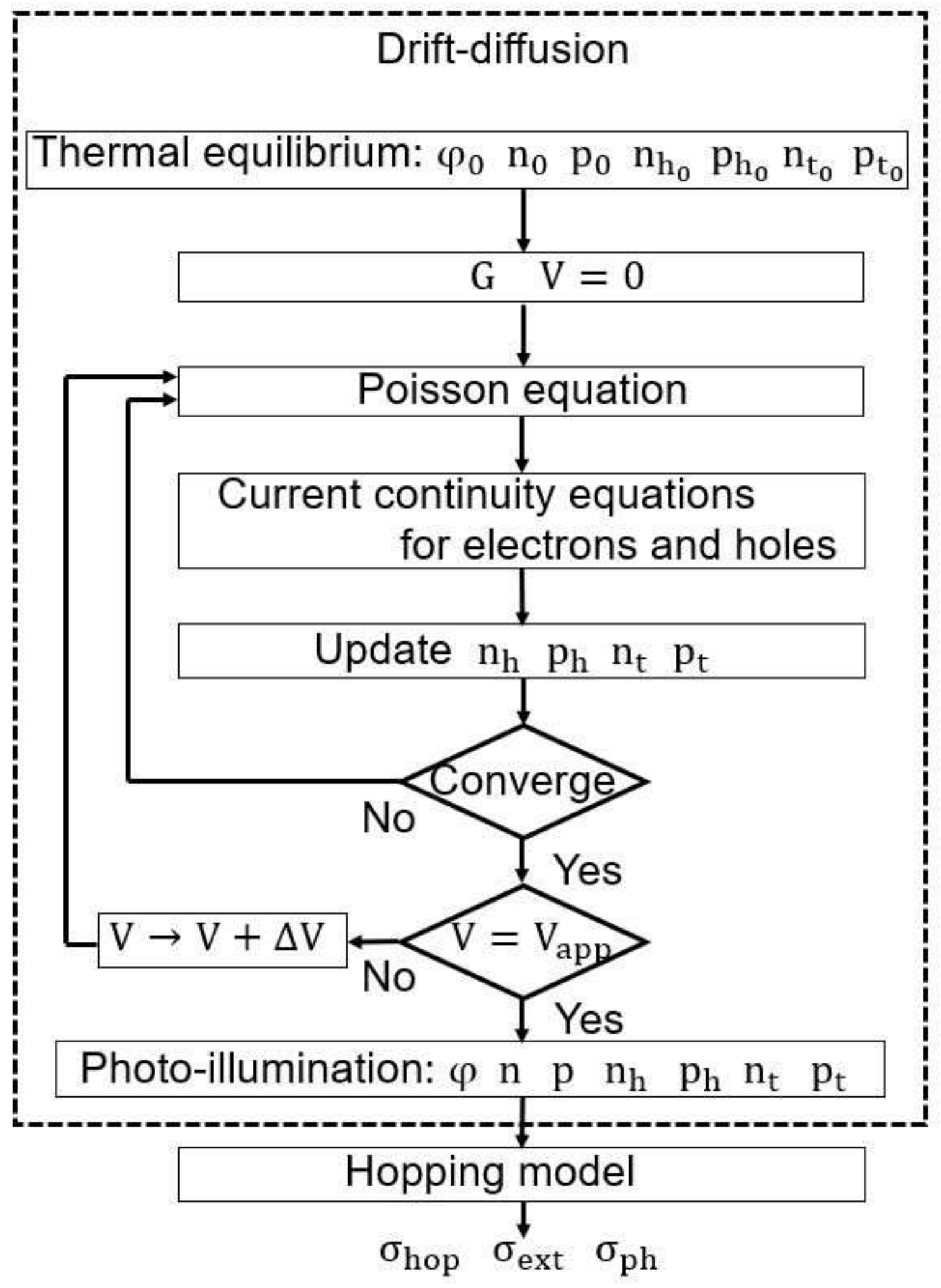

Figure 3

The flowchart of the present drift-diffusion simulator. All calculations are carried out in terms of the distribution functions derived by employing the principle of the nonequilibrium steady-state condition for the acceptor-like and the donor-like states self-consistently. Vapp (bias voltage) is $0 \mathrm{v}$ in this study. Furthermore, employing Mott's hopping models, the photoconductivity is derived with the results of the self-consistent drift-diffusion simulation. 


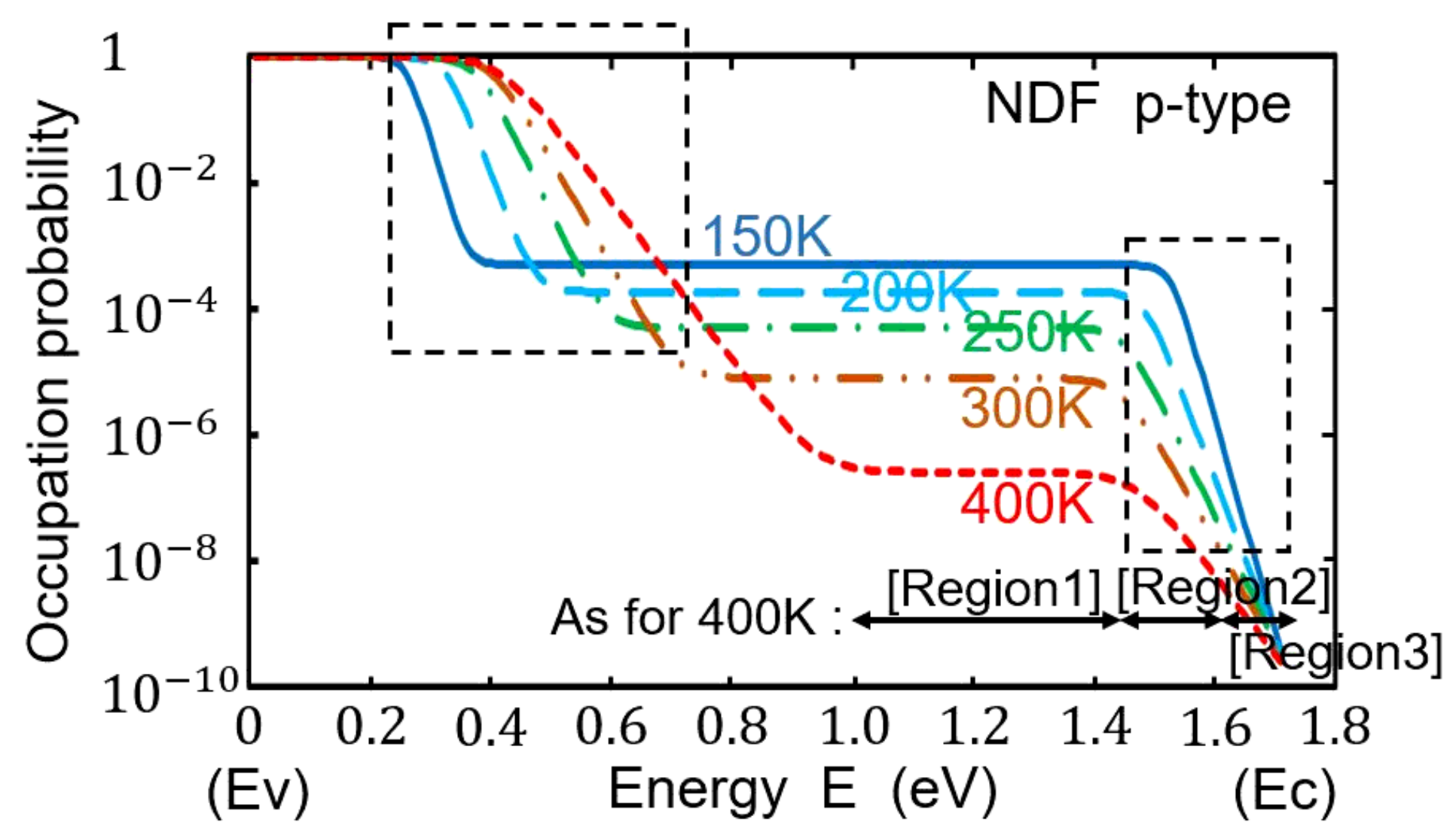

Figure 4

The nonequilibrium distribution functions (NDF) for the acceptor-like trap states as a function of energy for p-type at the temperature of $150 \mathrm{~K}, 200 \mathrm{~K}, 250 \mathrm{~K}, 300 \mathrm{~K}$, and $400 \mathrm{~K}$. These functions have stair shape structures: there are the energy regions to keep the values of the NDF constant ([Region1]). In terms of the temperature dependence of these functions, the values of these functions become smaller with increasing temperature (the negative temperature dependence) in [Region1], [Region2] and [Region3]. 


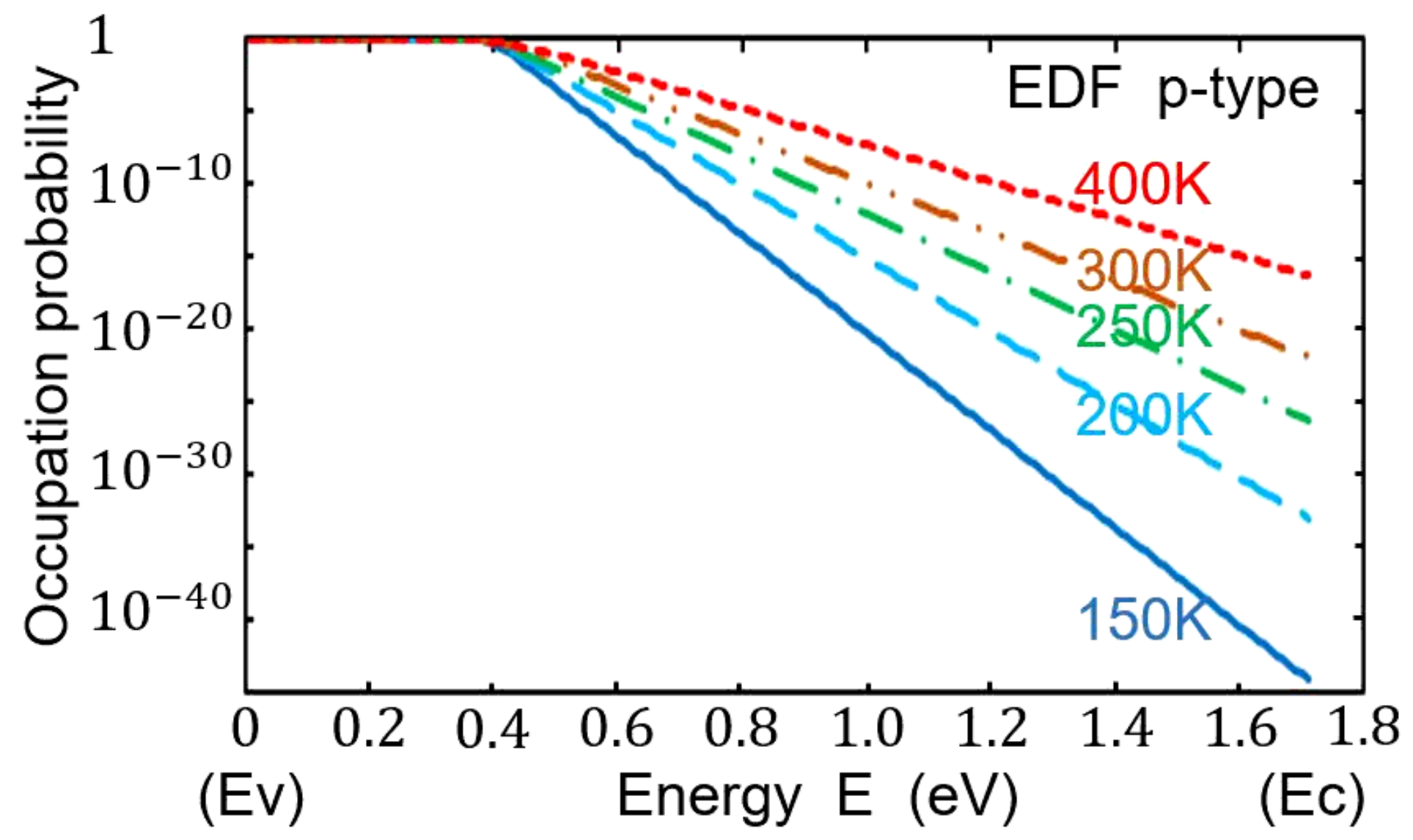

Figure 5

The equilibrium distribution functions (EDF) for the acceptor-like trap states as a function of energy for $p$ type at the temperature of $150 \mathrm{~K}, 200 \mathrm{~K}, 250 \mathrm{~K}, 300 \mathrm{~K}$, and $400 \mathrm{~K}$.
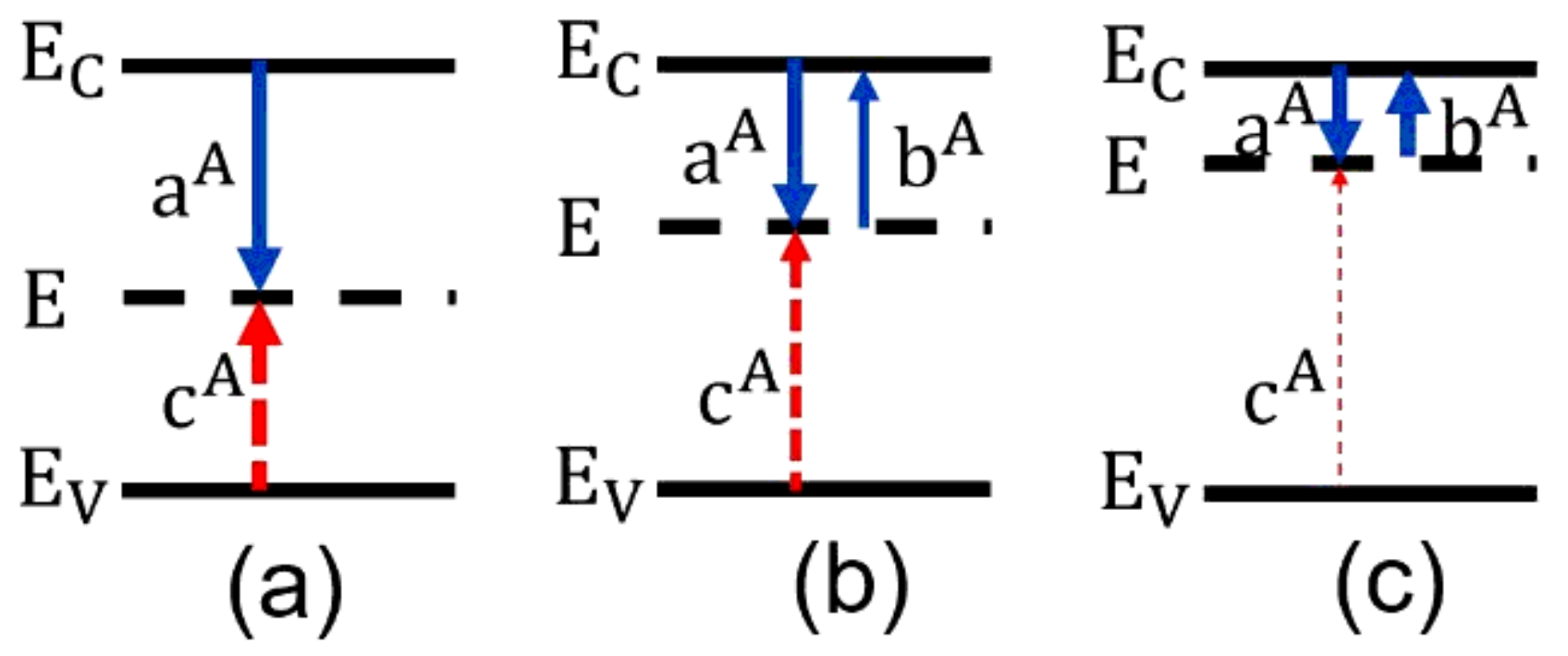

Figure 6

The schematics of the capture and emission processes via the acceptor-like states located at energy E. (a) The energy level $\mathrm{E}$ is far away from the conduction band and the valence band, the capture process of 
the electrons and that of the holes $\left(a^{\wedge} A\right.$ and $\left.c^{\wedge} A\right)$ are dominant. (b) The energy level $E$ gets closer to the edge of the conduction band, the electron emission process $b^{\wedge} A$ comes into play. (c) The energy level $E$ is remarkably close to the edge of the conduction band, the electron capture and the electron emission processes $\left(a^{\wedge} A\right.$ and $\left.b^{\wedge} A\right)$ become dominant, and the small difference between $a^{\wedge} A$ and $b^{\wedge} A$ balances with the hole capture process $\mathrm{c}^{\wedge} \mathrm{A}$. (a), (b) and (c) correspond to the energy level [Region1], [Region2] and [Region3] in Fig. 4, respectively.
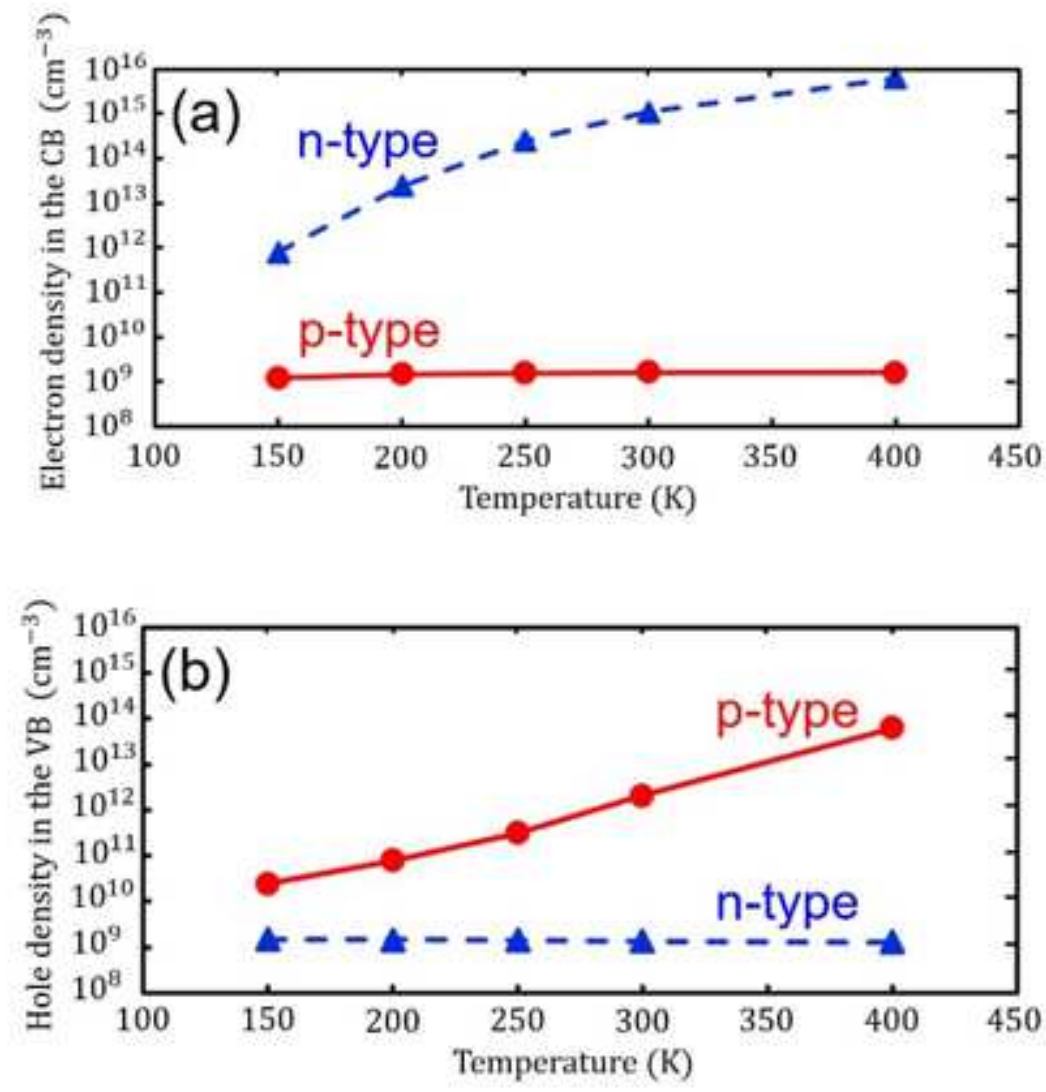

\section{Figure 7}

(a) The electron density in the conduction band as a function of temperature for $p$-type and n-type. (b) The hole density in the valence band as a function of temperature for p-type and n-type. The densities of the majority carriers produced by the photo generation and the thermal excitation in the bands increase exponentially with increasing temperature. On the other hand, the densities of the minority carriers in the bands are constant without the temperature dependence, because the densities of the minority carriers produced by the photo generation are much larger than those produced by the thermal excitation. 

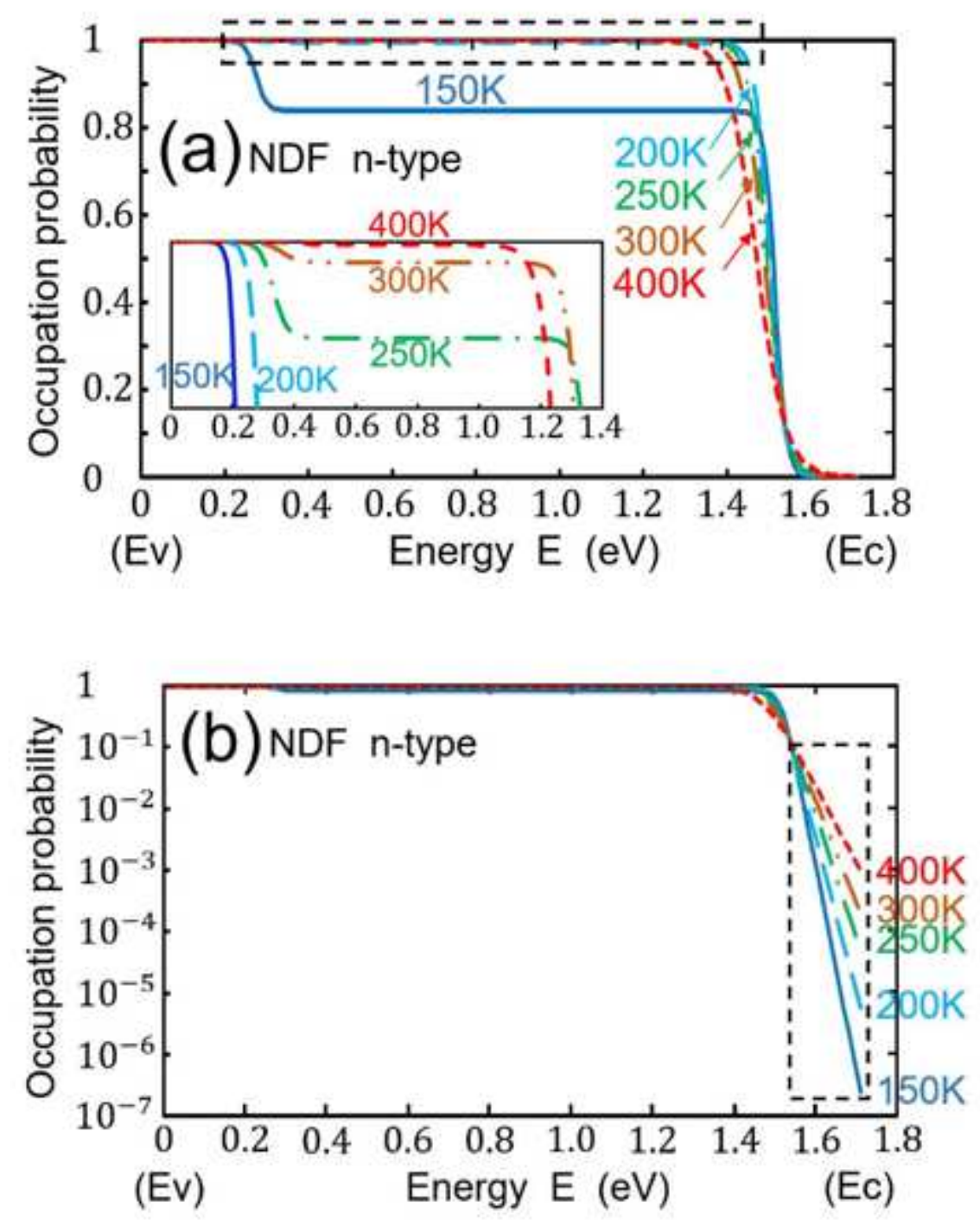

Figure 8

The nonequilibrium distribution functions for the acceptor-like trap states as a function of energy for $\mathrm{n}$ type at the temperature of $150 \mathrm{~K}, 200 \mathrm{~K}, 250 \mathrm{~K}, 300 \mathrm{~K}$, and $400 \mathrm{~K}$. (a) That is shown on linear graph; the inset is the enlarged figure in the area surrounded by the broken line. These functions have stair shape structures: there are the energy regions to keep the values of the NDF constant. The constant values become larger with increasing temperature (the positive temperature dependence). (b) That is shown on a semilogarithmic graph. In the vicinity of the $\mathrm{CB}$, the values of the NDF at respective energy level become larger with increasing temperature just like the EDF (the positive temperature dependence) as shown in the area surrounded by the broken line. 

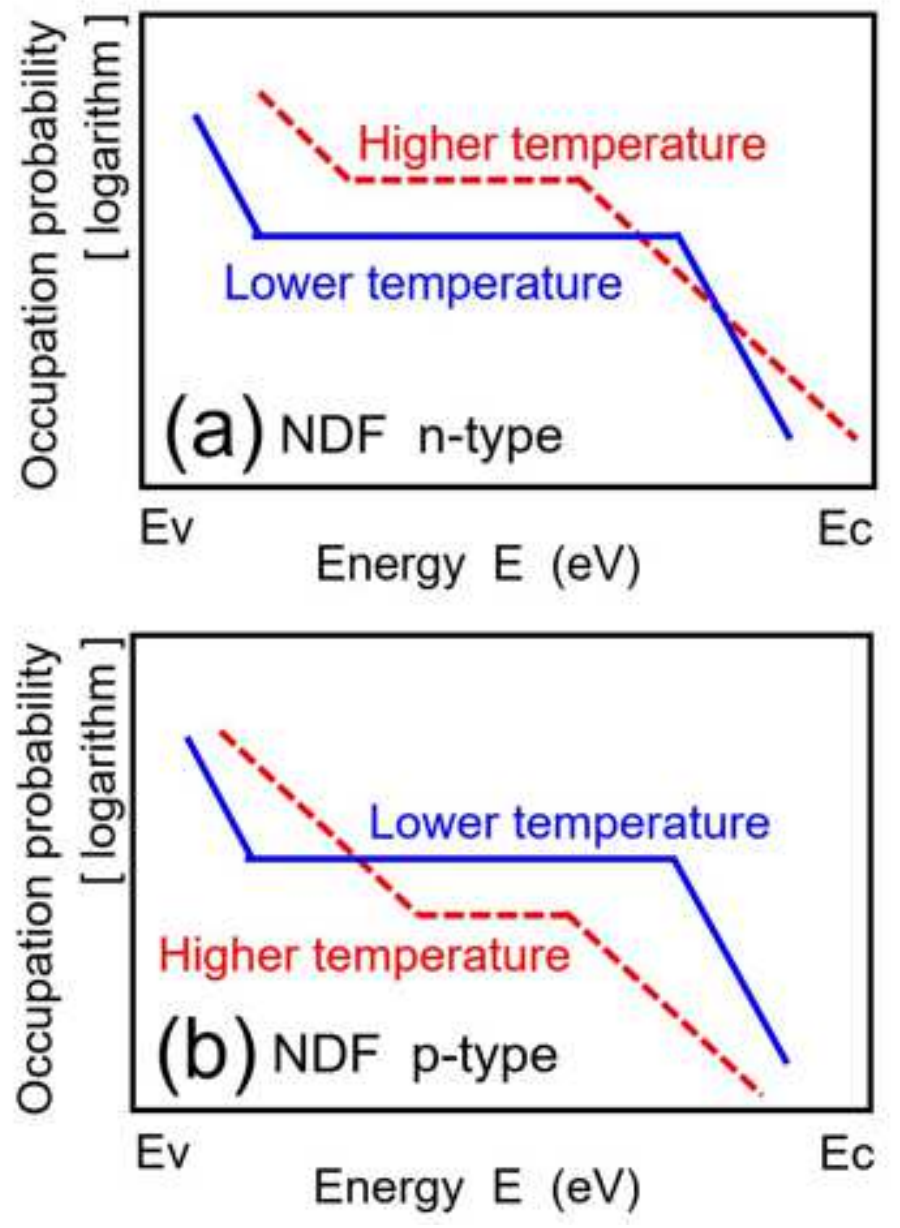

Figure 9

The explanatory figures to show the difference of the temperature characteristic between the NDF for ntype and that for p-type. (a) The NDF for n-type. (b) The NDF for p-type. The solid lines show the NDF for the lower temperature, and the broken lines show the NDF for the higher temperature. 

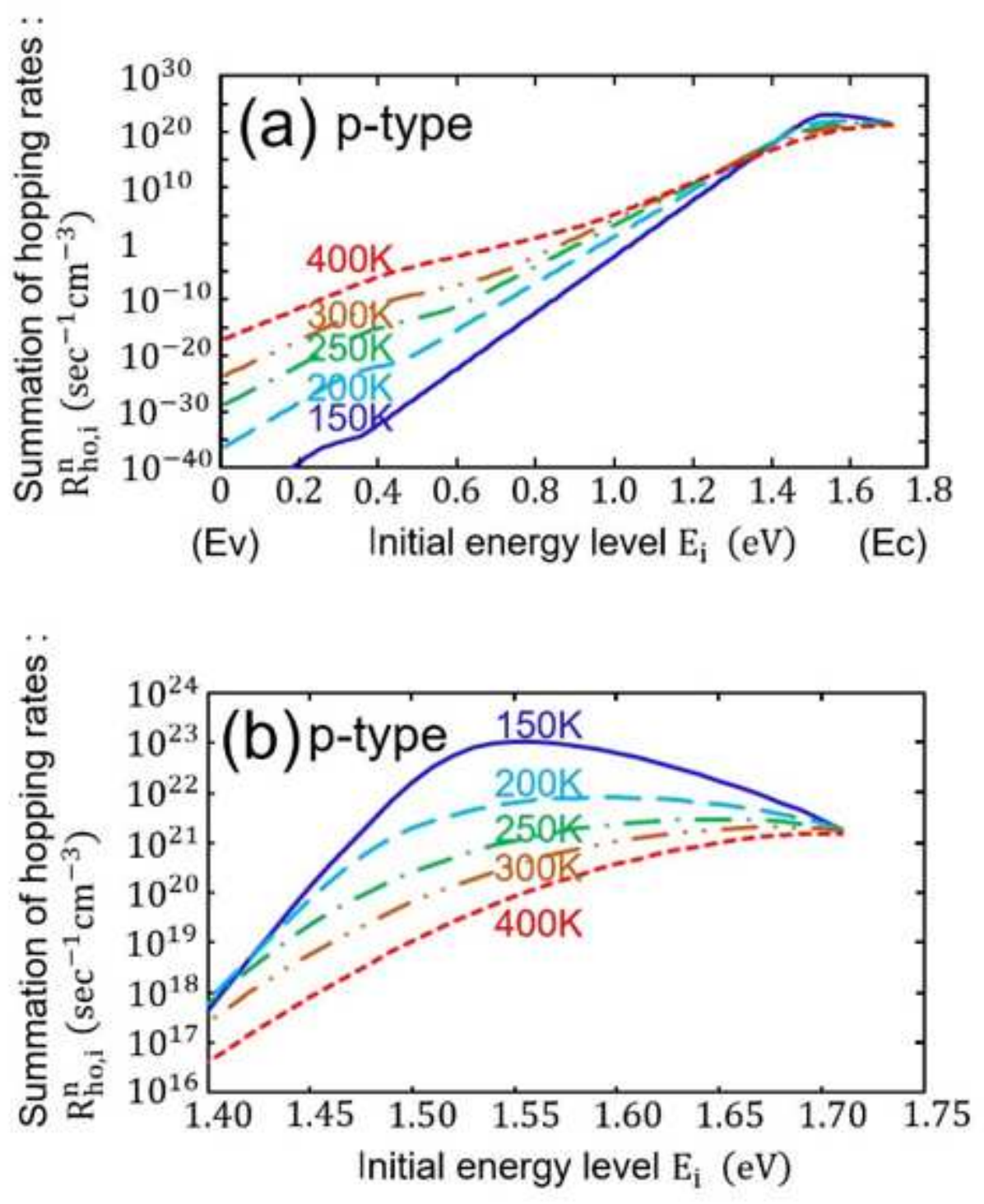

Figure 10

The summations of the hopping rates per unit volume $R_{-}(\text {ho,i })^{\wedge} n$ (Eq. (35)) for the trapped electrons in the acceptor-like band tail for p-type as a function of the initial energy level $E_{-} i$ at the temperature of $150 \mathrm{~K}$, $200 \mathrm{~K}, 250 \mathrm{~K}, 300 \mathrm{~K}$, and $400 \mathrm{~K}$. (a) That is shown in the whole energy level of the mobility-gap. It is found that $R_{-}(h o, i)^{\wedge} n$ near the conduction band is dominant. (b) That is shown in the vicinity of the conduction band; in terms of the temperature dependence of $R_{-}(h o, i)^{\wedge} n$, the values of $R_{-}(h o, i)^{\wedge} n$ become smaller with increasing temperature (the negative temperature dependence). 

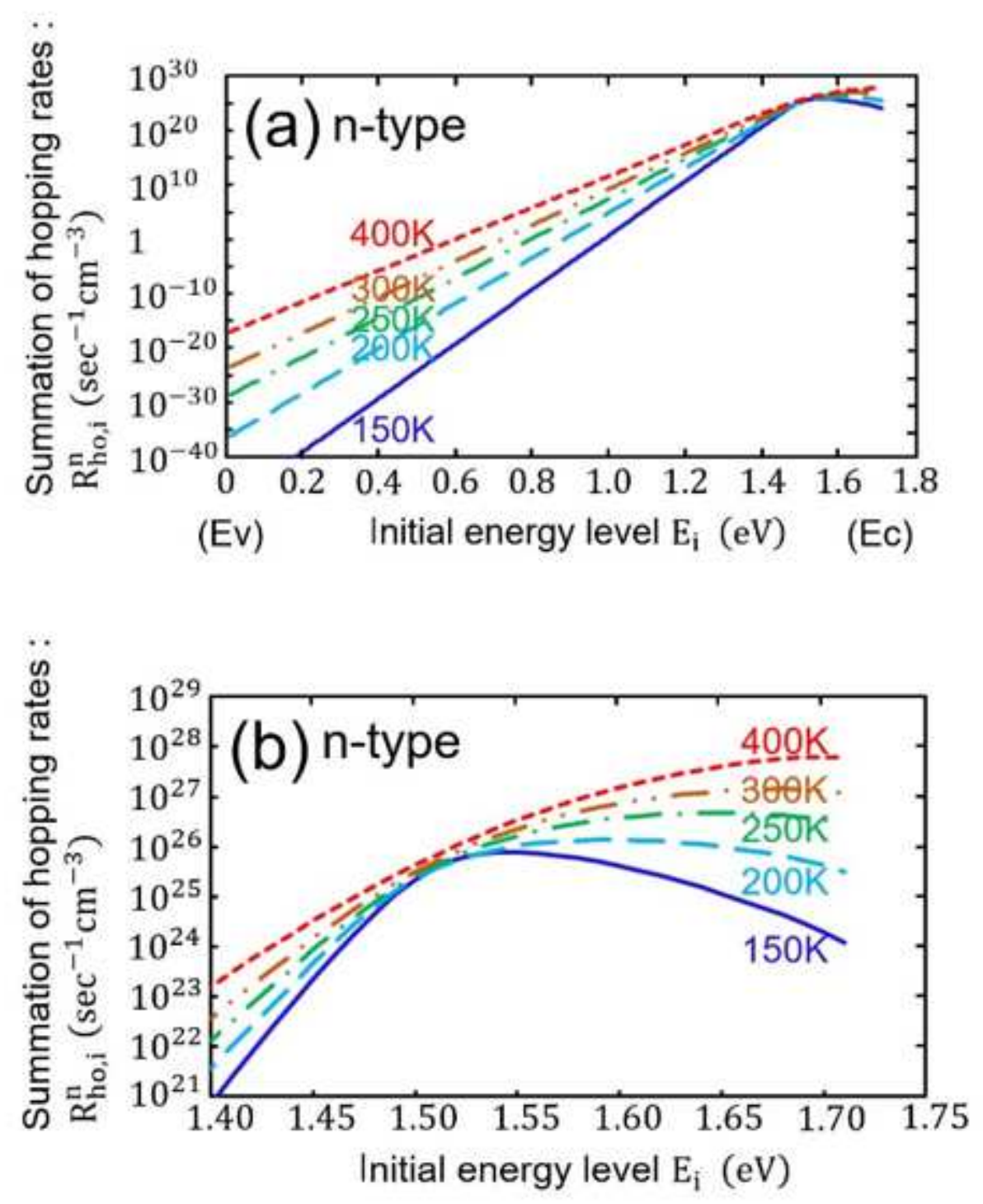

Figure 11

The summations of the hopping rates per unit volume $\mathrm{R}_{-}(\text {ho,i })^{\wedge} \mathrm{n}$ (Eq. (35)) for the trapped electrons in the acceptor-like band tail for n-type as a function of the initial energy level $E_{-} i$ at the temperature of $150 \mathrm{~K}$, $200 \mathrm{~K}, 250 \mathrm{~K}, 300 \mathrm{~K}$, and $400 \mathrm{~K}$. (a) That is shown in the whole energy level of the mobility-gap. It is found that $R_{-}(h o, i)^{\wedge} n$ near the conduction band is dominant. (b) That is shown in the vicinity of the conduction band; in terms of the temperature dependence of $R_{-}(h o, i)^{\wedge} n$, the values of $R_{-}(h o, i)^{\wedge} n$ become larger with increasing temperature (the positive temperature dependence). The temperature dependence of $R_{-}\left(h_{0}, i\right)^{\wedge} n$ for n-type is the reverse of that for p-type. 


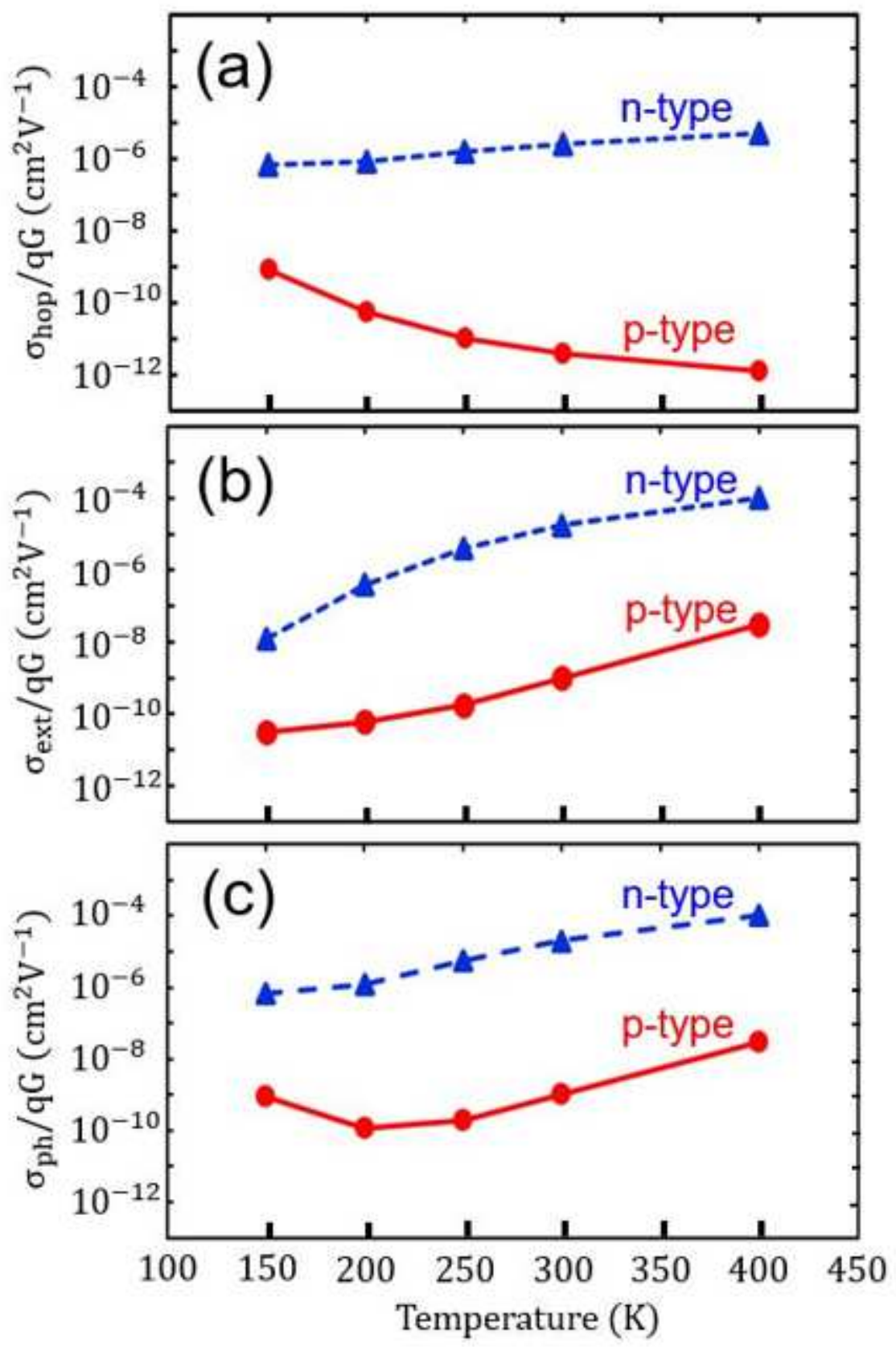

Figure 12

The calculation results of the photoconductivity as a function of temperature for $\mathrm{p}$-type and n-type. The photoconductivity is normalized by illumination intensity. (a) The electron hopping photoconductivity б_hop calculated from Eq. (38). (b) The band carriers' photoconductivity $\sigma \_e x t$, which consists of the photoconductivity for the electrons in the conduction band and that for the holes in the valence band, calculated from Eq. (39). (c) The total photoconductivity $\sigma \_p h$, which is the summation of $\sigma_{-}$hop and $\sigma \_$ext, calculated from Eq. (40). The thermal quenching is shown in the low temperature around $200 \mathrm{~K}$ for p-type. 